1

3

21

22

23

24

\title{
The Myth of Categorical Perception
}

\author{
Bob McMurray \\ Dept. of Psychological and Brain Sciences \\ Dept. of Linguistics
}

Dept. of Communication Sciences and Disorders

University of lowa

and

Haskins Laboratories

Contact

Bob McMurray

278 PBSB

Dept. of Psychological and Brain Sciences

University of lowa

lowa City, IA 52242

319-335-2408

Bob-mcmurray@uiowa.edu 
47

\begin{abstract}
Categorical perception is likely the single finding from speech perception with the biggest impact on cognitive science. However, within speech perception, it is widely known to be an artifact of task demands. Categorical perception is empirically defined as a relationship between phoneme identification and discrimination. As discrimination tasks do not appear to require categorization, this was thought to support the claim that listeners perceive speech solely in terms of linguistic categories. However, 50 years of work using discrimination tasks, priming, the Visual World Paradigm, and Event Related Potentials has roundly rejected this account. This paper reviews the origins and impact of this scientific meme and the work challenging it. It discusses work showing that the encoding of auditory input is continuous, not categorical, and describes the modern theoretical synthesis in which listeners preserve fine-grained detail to enable more flexible processing. This synthesis is fundamentally inconsistent with categorical perception. This leads to a new understanding of how to use and interpret the most basic paradigms in speech perception - phoneme identification along a continuum and has implications for understanding language and hearing disorders, development, and multilingualism. This challenges the field to develop new ways to describe and measure speech categorization.
\end{abstract}

\title{
Keywords:
}

Speech Perception; Categorical Perception; Categorization; Gradiency; Theories of Speech Perception 


\section{Introduction}

49 In the rich history of work on speech perception, Categorical Perception (CP) (Liberman et al.,

50 1957; Repp, 1984) is a breakout. It is one of a few findings to have made a lasting impact

51 outside of speech perception (Goldstone \& Hendrickson, 2010; Harnad, 2003) and its influence

52 has only grown (). CP is both an empirical phenomenon and a theoretical claim about the nature

53 of the representations that support speech perception. Empirically, it is observed whenever

54 discrimination (the ability to distinguish two stimuli) is affected by the presence of categories,

55 usually, when discrimination is poorer for two tokens in the same category than for tokens that cross the boundary (despite similar physical distance). Theoretically, it has led to claims that

57 perception is "warped" by the

presence of categories, and to the

broader claim that "analog or

continuous inputs are transformed

61 into quasi-digital, quasi-symbolic

62 encodings" (paraphrased from

63 Goldstone \& Hendrickson, 2010).

The problem is that both

65 claims are wrong. Speech

66 perception researchers who are

67 actively working on CP have known

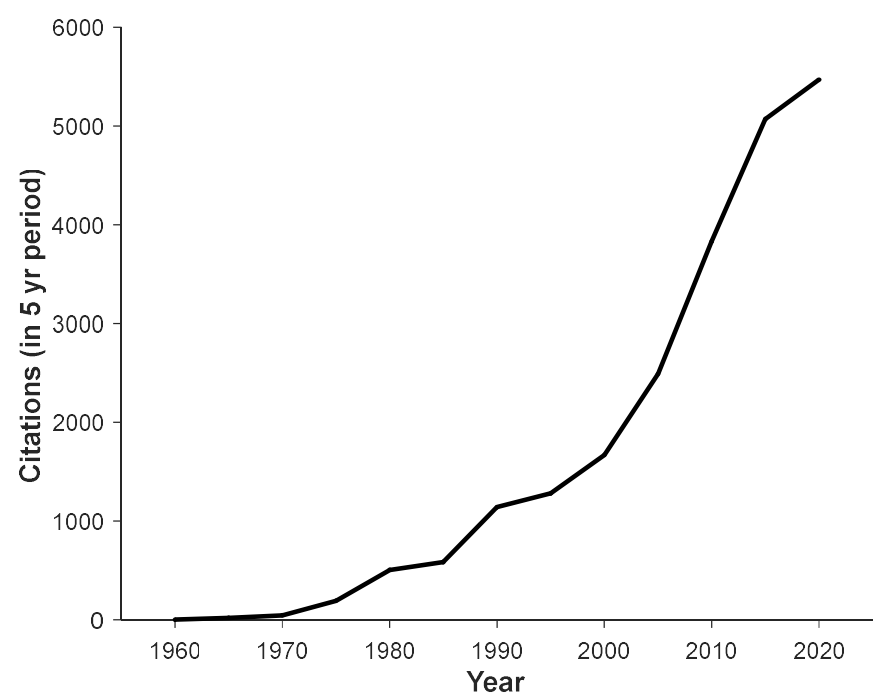

Figure 1: Number of new citations in consecutive 5-year periods starting in 1955. Search was conducted on Google/Scholar with the term "categorical perception"

this for many years (e.g., Massaro \& Cohen, 1983; Pisoni \& Lazarus, 1974). And yet long after the seminal work ruling out CP (both empirically and theoretically), CP continues to live on a sticky scientific meme ${ }^{1}$. It exerts a strong influence on the way speech perception is conceived

\footnotetext{
${ }^{1}$ Here, I don't mean a clever animated gif that bounces around the internet. Rather I use meme in Dawkin's original sense as a sort of sticky idea that persists because if fills a sociocultural niche, that is, an idea that has socio-cultural fitness.
} 
71 in broader work on language development (Maurer \& Werker, 2014), and communication

72 disorders (Serniclaes et al., 2004) and neuroscience (Chang et al., 2010); and it continues to 73 appear in other areas of perception (Beale \& Keil, 1995; Franklin et al., 2008; Freedman et al., 74 2001), and in other species (Freedman et al., 2001; Green et al., 2020). Even within speech, the assumptions made by categorical perception often implicitly shape ideas about what an ideal listener should be aiming for, or our understanding of why an impaired listener may differ. And

77 CP has left us with problematic and poorly understood tasks that are still used today for many 78 purposes besides categorization research.

Despite the substantial work countering the claims of CP there are few (if any) accessible reviews that explain why it is wrong, or that wrestle with the consequences of the failure of CP for both our understanding of speech perception, and for the methods we use to study it. This may be why CP still appears unchallenged in many textbooks, and why so many subfields of cognitive science do not appear to be aware of its demise. Thus, the purpose of this review is to directly lay out the evidence against $\mathrm{CP}$ and for alternative conceptualizations of speech categorization. By doing so I hope to challenge speech researchers to think deeper about the basic problems of perceptual categorization, and to spur our friends and colleagues in these other fields to do the same.

Given the vast quantity of work on $\mathrm{CP}$, this review is not exhaustive. I focus my review on work that has directly examined categorization in its "distilled" form (e.g., laboratory studies of monolingual typical adults) — my own area of expertise-and I lack the space and expertise to

91 offer more than a superficial discussion of other fields. This is not to claim that the work I chose

92 to focus on uniquely reflective of speech perception in general, nor that it offers greater insight

93 than the much larger variety of work in areas like sociolinguistics, speech pathology,

94 development, L2 learning, and other fields that contribute to speech perception as a whole. I

95 encourage the reader to consult those other literatures directly with these ideas in mind. Rather

96 I focus on this subfield because this is where the key ideas challenging CP originated, and 
where arose the largest body of literature that has directly sought to understand CP (rather than using it to make other insights). In many cases, it is this field where a critical breakdown in scientific story telling began.

This review is also a bit more blunt than is typical. The widespread view in the narrow community of researchers working on speech categorization is that $\mathrm{CP}$ is done, and there is no need to continue studying it: the apt titles of both Crowder's (1989) and Schouten, Gerrit and Van Hessen's (2003) papers say it all. However, the persistence of CP both in, and outside of, speech perception suggests the need for a more direct treatment. To be clear, I am not arguing that people do not categorize speech. In fact, the evidence for categorization is strong. Rather, as I lay out here, $\mathrm{CP}$ is a deeper claim about perceptual representations below the level of the category, and it is those claims that I attempt to refute here.

I start with a short overview of CP and its theoretical impact before discussing the evidence against it, and the modern theoretical accounts that require us to abandon CP. I then discuss areas of research where CP has led us astray and end with thoughts for the future.

\section{Categorical Perception}

Categorical perception (CP) simultaneously is defined as both an empirical phenomenon and a theoretical view of speech perception.

\section{A. Empirical Definition of CP}

$\mathrm{CP}$ is typically assessed in experiments in which listeners hear tokens from a continuum of speech sounds (e.g., spanning /b/ to /p/ in small steps). CP requires tasks that assess labeling (is each token a /b/ or a /p/?) and discrimination (are two tokens the same or different?). It is generally though to observed when three conditions are met: 1) listeners must show a sharp labeling function (Figure 2, dashed line): as the speech continuum advances, there is a sudden shift in the categorization; 2) listeners should be poor at discriminating tokens from the same 
category; 3) they should be good at

discriminating tokens that cross the boundary (solid line).

The first criterion is difficult to assess. Given a two alternative forced choice task, the psychophysical

function cannot mathematically be

linear: a listener cannot choose any

category more than $100 \%$ of the time.

Thus, the function must reach

asymptotes at either end, and even it if

appears linear this is probably an

artifact of where the endpoints are set.

Thus, the classic sigmoidal curve

associated with $\mathrm{CP}$ in fact, means very

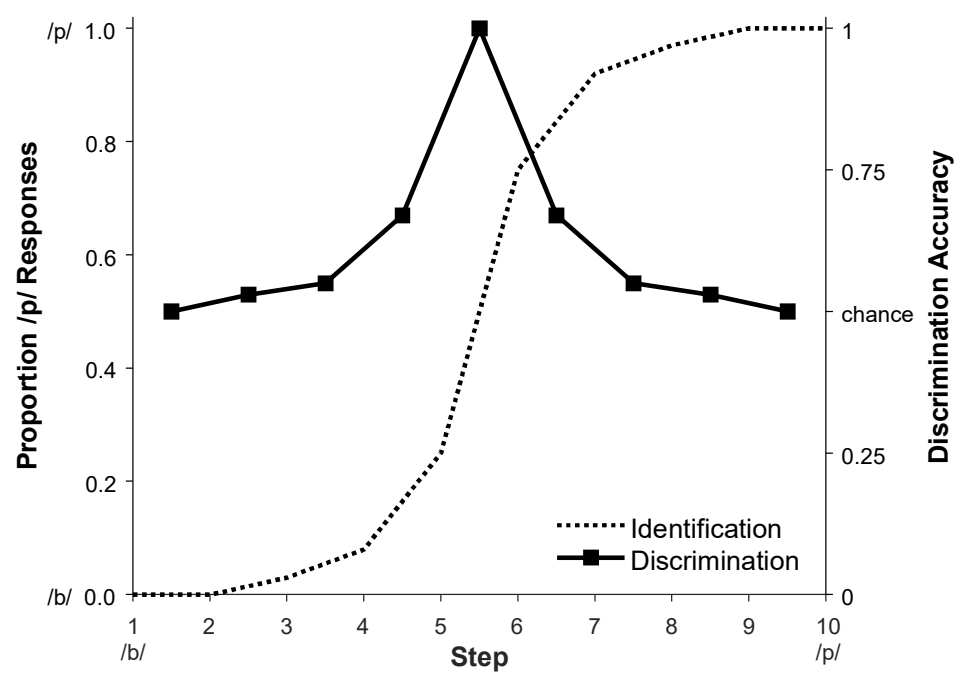

Figure 2: Canonical results of a categorical perception experiment. On the $X$ axis, step refers to step from a speech continuum, where step 1 might be a prototypical $/ \mathrm{b} /$ and step 10, a prototypical / $/ \mathrm{p} /$. Identification (dashed line, left axis) is coded as the proportion of responses that match one of the endpoints. Here, 0 is /b/ and 1.0 is $/ \mathrm{p} /$, so that $0 \%$ responding indicates the participant consistently heard /b/. Discrimination is assessed between neighboring points (e.g., between step 1 and 2 , 2 , and 3 , and so forth). The peak indicates that discrimination is better between steps 5 and 6 (spanning the boundary shown by the identification results) than between steps 1 and 2 (both /b/).

little other than that subjects had to make a forced choice task from a continuum. It is not and can never be evidence for CP. Moreover, the steepness of the function is similarly problematic. While the strongest form of CP claims a perfect step function (a transition of $100 \%$ between the smallest possible distance), this has never truly been tested (e.g., with adaptive approaches that use increasingly smaller steps). Instead, researchers generally informally evaluate the slope and declare it steep. But the slope is in part related to the step size and the range of the continuum (Brady \& Darwin, 1978; Rosen, 1979). By selectively testing a narrow range (or a small step size), a researcher could in principle create a sharper or shallower transition.

Moreover, across different speech cues, step is often meaningless and incommensurate (how does one compare a $5 \mathrm{msec}$ Voice Onset Time [VOT] difference for a voicing continuum to a 40 Hz F1 difference in a vowel continuum), and there have been few attempts to standardize them 
149 (see Box 1 for thoughts). Thus, for all intents and purposes, the first criterion cannot be 150 rigorously evaluated.

The second and third criteria are often treated similarly loosely. Some have caricatured $\mathrm{CP}$ as requiring no above chance within-category discrimination. It has been pointed out that even the original demonstration of CP (Liberman et al., 1957) did not observe this (and in fact it found robust discrimination within the /g/ category). However, this characterization is not 155 baseless. Indeed, Liberman et al. (1961) writes in the abstract to the second CP paper: "The 156 perception of these stimuli is essentially categorical in that $S$ can hear no differences among the 157 stimuli beyond those that are revealed by the phoneme labels he applies to them." Nonetheless, 158 this high bar is not conventionally required to establish CP and many studies have collapsed the 159 second and third criteria to the relative difference (discrimination is better for tokens that span a category boundary than those that lie within).

A more rigorous approach than comparing within- and between-category discrimination,

162 is to directly predict discrimination from the labeling responses using formal models (Gerrits \& 163 Schouten, 2004; Liberman et al., 1957; Schouten et al., 2003). Such models start from the 164 strong assumption that listeners perceive speech solely in terms of labels. Under this assumption, discrimination should be at chance if two tokens are labeled the same $100 \%$ of the 166 time, and should increase if labeling of those tokens differs. The predicted discrimination 167 function is generated from an individual subject's labeling and can then be compared to the 168 obtained discrimination. To the extent that they match, CP can be claimed. To the extent that 169 discrimination exceeds predictions, some within-category sensitivity must be present. Again, 170 perfect predictability need not be found to claim CP (e.g., identification and discrimination do not 171 need to be $100 \%$ isomorphic), any influence of identification on discrimination is sufficient. Using variants of this definition based on identification and discrimination, CP has been

173 shown across a variety of speech contrasts including place of articulation (Liberman et al., 174 1957) and voicing (Liberman et al., 1961) in stop consonants, vowels (Van Hessen \& Schouten, 
1999) (though see Fry et al., 1962), liquid contrasts like I/r (Miyawaki et al., 1975), as well as the fricative/affricate contrast (Cutting \& Rosner, 1974).

This definition of CP has not been consistently applied. First, many studies and presentations use the term Categorical Perception to refer to a steep labeling function alone (even as steepness itself is fraught); others use CP to refer to any experiment that asks subjects to label tokens from a continuum even with no discrimination measure. This is wrong. We must end this rhetoric. CP invokes a specific theoretical view of speech, which as I argue shortly, no longer holds. Thus, by invoking CP as a sort of shorthand for this approach, researchers are unintentionally making stronger claims about perception than they intend. Even as a purely methodological term, these studies are simply measuring categorization—not CP.

Second, in other cases, CP is sometimes claimed on the basis of discrimination data alone in the absence of a labeling task. This is commonly the case in the infant literature where most infant measures only permit a measure of discrimination (see McMurray, in press, for a review); however, it also applies to neuroimaging techniques that use approaches such as the mismatch negativity in EEG or representational similarity analyses to construct a neural version of discrimination, but without any corresponding neural version of identification. Such practices risk affirming the consequent: we know a particular profile of discrimination is consistent with $\mathrm{CP}$; $\mathrm{CP}$ requires categorization; therefore, when that profile is observed, we can assume categorization. However, as I describe shortly, the evidence against CP as an empirical description of adult speech perception is now substantial and in retrospect the evidence for it appears weaker than one might thing. If we remove CP from the derivation chain, it is not clear what can be concluded from discrimination alone.

\section{B. Theoretical Implications of CP}

$\mathrm{CP}$ is more than an interesting empirical phenomenon-it makes deep theoretical claims about speech. In fleshing out these claims, CP starts from a model of speech recognition with (at 
201 least) two levels of analysis

202 (Figure 3). First, the input is

203 mapped onto some form of

204 auditory encoding that reflects

205 the continuous nature of

206 acoustic cues like the spectral

207 peak of a fricative, formant

208 frequencies of a vowel, or the

209 VOT of a stop. For

210 convenience, I refer to these as

211 phonetic cues. This assumption

212 is not intended to make strong

213 claims as to the validity of any

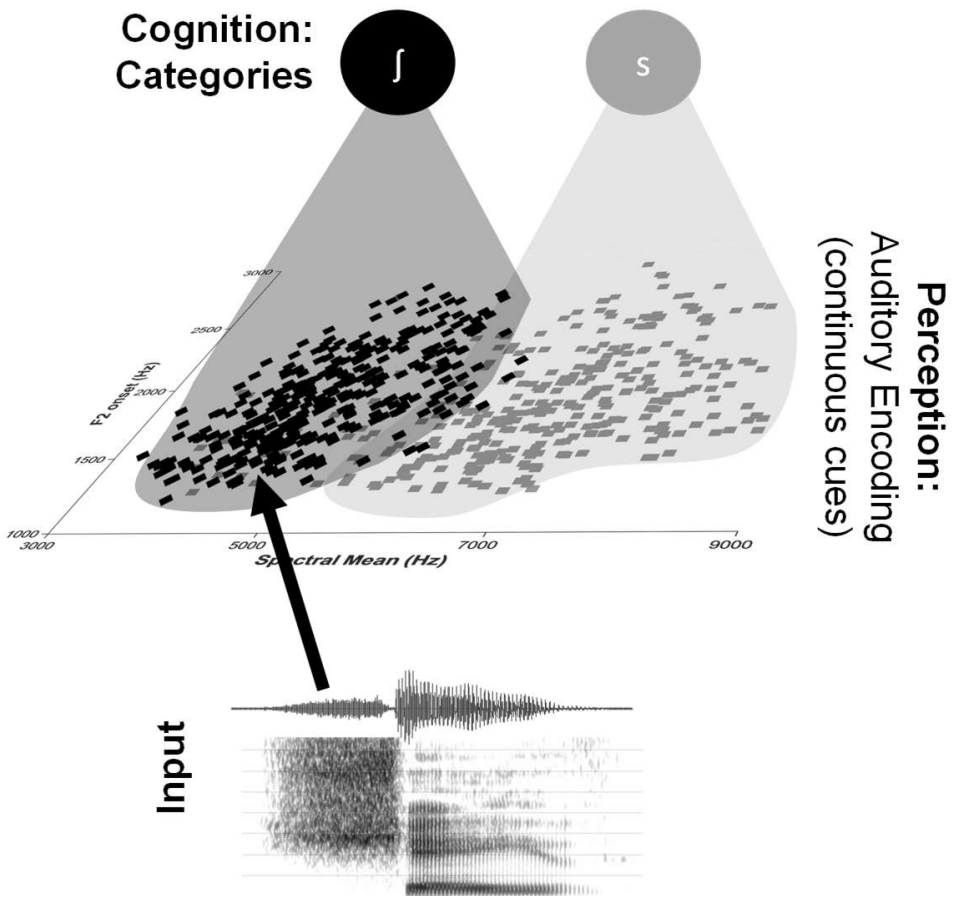

Figure 3: A rough conceptual model of the speech chain assumed by CP. Speech input is first mapped to a continuous auditory encoding that represents something like continuous cue-values. This space is then carved into categories.

214 specific cues, and most likely this level is a m ultidimensional representation across multiple

cues. Critically, this level of representation is continuous: at some level it preserves the gradient nature of the input in a way that reflects acoustic similarity. Second, cues are then carved into categories like phonemes, features, or words. In the framing of $\mathrm{CP}$, perception, refers to the continuous auditory encoding, and categorization is a later cognitive process operating on perception. This is an important contrast from the more modern (looser) use of these terms where perception might include a wide range of processes, including categorization. What are the consequences of this framing for the empirical paradigm of CP?

222 Discrimination tasks do not require labeling. For example, in the ABX task used by Liberman et 223 al. (1957), listeners hear two sounds that are different (the A and B sounds) followed by a third 224 that matches one or the other. They indicate which of the first two matches the third. For 225 example, if they heard "ba pa pa", the correct answer is \#2. In principle, a listener could perform this task this without categorical labels; if they heard ba ba $\underline{b a}$ (where the underline indicates a 
227 lower pitch), they could correctly identify the second as the match. Thus, discrimination tasks 228 were presumed to tap the auditory level of encoding.

If we assume that discrimination only reflects the auditory level, CP makes a bold claim: that the fundamental encoding of the continuous cues is altered or warped by perception: tokens from different categories are heard as further from each other, and tokens from the same category are closer in perceptual space. This violated Weber's law (that perceptual distance is a monotonic function of stimulus distance), as perception is "warped" toward a more discrete 234 representation of speech. In some ways this can be seen as a form of perceptual constancy 235 despite differences in the phonetic cues, stimuli are perceived as the same (as long as they are within the same category). The perceptual warping reflected by CP was a view that was well suited for its moment in the history of cognitive science for two reasons.

First, at this time, work at Haskins Laboratories, the Massachusetts Institute of Technology, and Brown University was starting to flesh out the problem of lack of invariance (Blumstein \& Stevens, 1979; Delattre et al., 1955): the idea that variability due to talker differences, speaking rate, and coarticulation makes it such that there is no one-to-one mapping 242 between an acoustic form and an underlying category. This is illustrated in Figure 4 which 243 shows a set of measurements of /s/ and /// across multiple vowels and talkers by Jongman et al. 244 (2000). Figure 4A shows the raw values along with the boundary computed from a discriminant 245 analysis. Note the considerable overlap between the categories; this is created by the fact that 246 this corpus contains 20 different talkers and 6 different coarticulatory contexts (the neighboring 247 vowel). Figure 4B shows these same measurements transformed by a simple version of CP 248 which warps these measurements to enhance between-category distance and minimize 249 differences within the category. Here, tokens to the left of the discriminant boundary are shifted 250 leftward and upward (the reverse for those on the right), and the variance within each category 251 was halved. This illustrates how CP could alter the auditory representation to highlight 252 categories (I discuss $4 \mathrm{C}$ and $\mathrm{D}$ later). The critical idea is that CP seems to make the problem of 
253 invariance less challenging. CP

254 does not solve this problem-

255 in fact the few tokens on the

256 other side of the boundary are

257 now misclassified in even more

extreme ways. However, it may
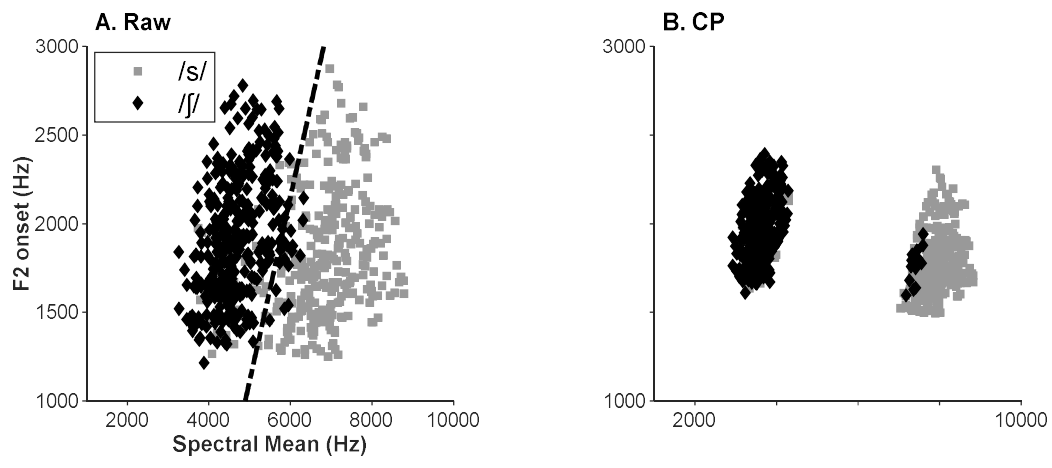

make the initial percept more

robust against later noise.

261

More broadly, at the time it was

262

discovered, the empirical

263

evidence for CP implied that

listeners may be equipped with

C. CP $-b / w$ only
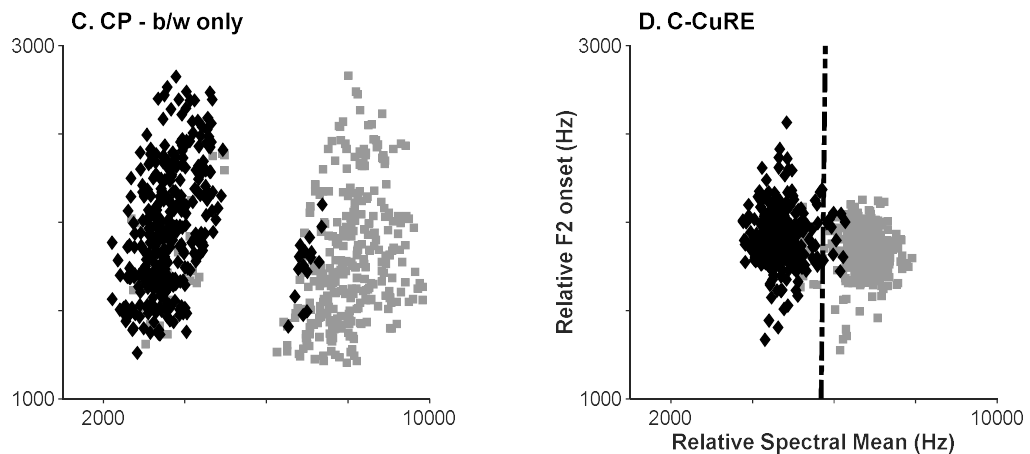

Figure 4: A) Spectral mean and F2 onset frequencies for /s/ and /J/ from Jongman et al. (2000). The line represents a linear discriminant analysis that can separate the categories at about $92 \%$. B) A simulation of CP generated by assuming the linear boundary in $\mathrm{A}$, and then doubling the distance between tokens on each side of the boundary and halving the variation within each side of the boundary; C) A simulation of CP generated by only increasing the distance, without adjusting within-category variation. D) Same data after compensating for talker and vowel differences using the regression approach of C-CuRE (McMurray \& Jongman, 2011); the discriminant analysis achieves $96.6 \%$ accuracy.

Second, in the late

1950s, the cognitive revolution was underway. In fact, Chomksy's (1959) review of Skinner's

271 (1957) Verbal Behavior, which in part sparked this revolution, was published shortly after the

272 seminal CP paper (Liberman et al., 1957). The cognitive revolution argued for a view of

273 cognition that was built on symbolic operations. CP supported this symbolic view by arguing for

274 the existence of mechanisms that rapidly transduce the continuous signals into discrete

275 categories. Moreover, as research on CP developed, it became linked to other key ideas in the

276 cognitive revolution: for example, it may be innate and specific to speech (Liberman et al.,

277 1967). Supporting this special mode of speech perception, there were early demonstrations that

278 CP could be observed with speech, but not non-speech sounds that capture similar acoustic 
relationships (Liberman et al., 1961) (and see Cutting \& Rosner, 1974, for a nice review of these early attempts to find CP in non-speech analogues).

As I describe, the place of CP in arguing for "speech is special" claims did not hold up: it appears with a variety of non-speech auditory stimuli, in many other domains, and in other species. Nevertheless, the broader theoretical claim has persisted: that the perception of continuous acoustic representations is warped by higher-level language knowledge and in a way that support categorization. A variety of mechanisms have been posited to account for CP. One early prominent account is that $\mathrm{CP}$ derives from natural discontinuities in the auditory system itself (e.g., discontinuities in perceiving any time difference near $20 \mathrm{msec}$, the typical VOT boundary); this was supported by work showing CP in non-human animals (Kuhl \& Miller, 1975), and by work with new non-speech analogues that capture similar acoustic dimensions (e.g., tone-onset-time as an analogue of VOT) and that showed similar discrimination peaks to $\mathrm{CP}$, and boundaries at similar locations to those of speech continua (Cutting \& Rosner, 1974; Pisoni, 1977). Auditory discontinuities were also supported by later electrophysiological work using direct recordings from auditory cortex (Steinschneider et al., 1999). Under this view, languages evolved to take advantage of these inherent discontinuities.

The discontinuity approach, however, was unlikely to fully explain the phenomenon. First, this work focused on a handful of phonetic contrasts (largely voicing and place of articulation in stop consonants) and it is not clear if auditory discontinuities could be found for many other phonemic contrasts needed to fill out a language. Moreover, languages vary in where they place their boundaries, and it is not clear if discontinuities would line up crosslinguistically. If CP derives from such discontinuities and if CP is invoked as a general theory of speech perception, these facts must be explained. Second, some empirical results with nonspeech analogues have not held up to scrutiny and may derive from methodological confounds (c.f., Rosen \& Howell, 1981). Third, animal models are problematic as animals must be extensively trained to do these tasks. CP in animals could be an artifact of learning, not an 
inherent property of the auditory system.

One compelling alternative account of CP was that the auditory space is warped by learning. Overtraining on particular classes of sounds (over development) has led the system to devote more representational or neural space to certain subregions of the acoustic space (Guenther \& Gjaja, 1996; Liberman et al., 1961). This still posits the same fundamental discontinuity in the auditory system that is the basis of $\mathrm{CP}$, but its origin is learning. In some ways, this is more parsimonious as it can in principle apply to any speech sound or to any set of boundaries used by a given language. It is supported by work, for example, showing that CP peaks align closely with the language of the listeners: Japanese listeners, for example, show no discrimination peaks in an ///-/r/ continuum while English speakers do (Miyawaki et al., 1975); similarly with non-speech sounds, the degree of CP for a major/minor continuum is related to the amount of musical experience (Howard et al., 1992). This has been instantiated in neural models (Gauthier et al., 2007; Guenther \& Gjaja, 1996) and empirically examined with MRI (Guenther et al., 2004), suggesting plasticity in neural "maps" (of the continuous acoustic space) such that more representational space is given to categories.

In a similar vein, CP may derive from an interactive feedback loop (Anderson et al., 1977; McClelland \& Elman, 1986) (and see Lupyan, 2012 for extensve discussion of this idea in visual $\mathrm{CP}$ ); that is, as the system settles on one choice, activation at the category level feeds back to earlier levels of analysis, aligning the perceptual representation with the category decision. This can be seen as an indirect effect of learning (since the initial decision is grounded in learning), but one that doesn't alter the inherent structure of the auditory representation; rather it is functionally altered in real time as perception and cognition align.

Whatever the theory, the strong assumption of $\mathrm{CP}$ is that the perceptual space (defined by $\mathrm{CP}$ as the pre-categorical, auditory encoding) is at least partially discontinuous. The depth of this assumption is perhaps illustrated by the TRACE model of speech perception and word recognition (McClelland \& Elman, 1986). This model was built on fundamentally different 
331

332

333

334

assumptions about cognition from the information processing view: it rejects both the need for discrete symbols, and the specialness of speech. Yet McClelland and Elman still felt the need to extensively explain CP (pp. 43-50) as a product of competition. This has left a consensus reflected in virtually all psycholinguistics and phonetics textbooks -- that whatever the mechanism, the auditory encoding does not faithfully reflect the input and is warped in a way to rapidly transduce auditory input to something that is quasi-discrete.

\section{Beyond Speech}

This formulation has now extended beyond speech. CP has been shown in complex auditory domains such as musical categories (Howard et al., 1992; Locke \& Kellar, 1973). It has appeared in several domains of visual perception including lower-level visual cues like color (Bornstein \& Korda, 1984) and line orientation (Quinn, 2004), and to higher-level visual stimuli like faces (Beale \& Keil, 1995), facial emotion (Hess et al., 2009), and object categories (Newell \& Bülthoff, 2002). It has been shown in the tactile and haptic (touch) perceptual systems (Gaißert et al., 2012; Knight et al., 2014). And it has even been observed in students' judgements of p-values (Rao et al., 2021), a domain which in which perception is hardly relevant! It has been shown in a variety of species, often for species-relevant signals (e.g., birdsong) (Lachlan \& Nowicki, 2015; May et al., 1989; Wyttenbach et al., 1996), suggesting a general adaptation that is a consequence of learning meaningful signals.

This work outside of speech has enriched the debate. It would be surprising (though not impossible) if fundamental perceptual discontinuities appeared in all of these domains, supporting theories based on learning and/or feedback. Moreover, this research has revealed unequivocally that $\mathrm{CP}$ is at least a partially learned phenomenon (also consistent with the feedback hypothesis). For example, it appears for familiar but not unfamiliar faces (Beale \& Keil, 1995) and it can be invoked by learning new faces (Goldstone et al., 2001). It appears in musical chords only for musically trained people (Howard et al., 1992). And CP in color vision is 
357 linked to the specific color terms of the participant's language (Roberson et al., 2000), and 358 developmentally linked to learning the color words (Franklin et al., 2008).

In these domains, CP has often been used to support similar viewpoints as in speech:

that a given domain is handled by a system that transduces a continuous sensory space to categories (Knight et al., 2014), often by specialized mechanisms. For example, inverted faces are often used in studies of whether there are face- specific processing modules and CP is reduced (or eliminated) in inverted faces (McKone et al., 2001) (though see Levin \& Beale, 2000). Similarly, hemispheric asymmetries are often invoked to argue for a role of language, and color $\mathrm{CP}$ is stronger when stimuli are presented to the right visual hemifield (Franklin et al.,

366 2008), which projects most strongly to the left hemisphere. In fact, the power of the CP metaphor for rapidly transducing a noisy signal to an abstract quasi-symbolic code has led to

368 the broader claims that it is foundational to cognition (Harnad, 2003)!

\section{But...}

371 CP appears to offer a compelling package. The work reviewed so far suggests CP is a domain372 general (and maybe species-general) mechanism in which learning warps the perceptual space 373 in a way that may help deal with the substantial variability in the input. Unfortunately, it is wrong.

The empirical evidence for $\mathrm{CP}$ is not strong, and the theoretical views it inspired is

376 fundamentally inconsistent with modern theories of speech. This is not to rule out categorization 377 as an essential aspect of speech perception. Rather, what's wrong is the deeper claim about 378 auditory/perceptual encoding. In making this argument I address two claims. First, is the direct 379 claim of CP for sensory warping (Section III). Second, is the broader claim about the functional 380 goal of transducing speech to quasi-discrete categories (Section IV). While this latter point is 381 direct evidence against $\mathrm{CP}$, it is evidence against the broader view of speech rooted in CP. 


\section{III. $\quad$ CP is an inaccurate empirical description of perception}

Compelling evidence against $\mathrm{CP}$ as an empirical phenomenon is found in several lines of work.

\section{A. $\quad C P$ is not universal across speech sounds}

387 From the beginning, it was known that CP was not observed for all speech contrasts. Early 388 studies with vowels did not show CP (Fry et al., 1962) (though Van Hessen \& Schouten, 1999, 389 later showed that you can with the right stimulus manipulations), with no difference between 390 within- and between-category discrimination and fricatives show reduced CP (Healy \& Repp, 391 1982), less of a difference than would be expected. More recent work has shown noncategorical perception for Mandarin tones (Francis et al., 2003). Idiosyncratic findings of CP are also seen in vision: Newell and Bülthoff (2002) report CP across only a subset of visual objects.

It might be easy to chalk these cases up as exceptions to a general principle of CP. This is problematic for three reasons. First, the number of speech sounds for which CP has been shown is not large. Much of the work has examined only stop consonant place and voicing.

397 There are large swaths of the phonetic space that have not been systematically examined: many vowel contrasts, nasals, some approximants, and some manner of articulation distinctions. Consequently, it is not clear if the contrasts showing non-categorical perception are the exceptions, or if the cases showing CP are! Second, if CP derives from a learned warping or feedback mechanism (which appear to be the dominant accounts at the moment), why do these mechanisms not operate for all sounds? It's not clear how the general principles invoked by CP interact with the specifics of the phonetic cues to give rise to such idiosyncratic effects. Finally, these findings are inconsistent with the theoretical view that $\mathrm{CP}$ reflects adaptations designed to 405 help cope with the problem of lack of invariance. Vowels, fricatives and tones all show some of 406 the strongest contextual dependencies due to talker and coarticulatory differences. These are 407 the contrasts for which lack of invariance is arguably a more serious problem. If CP is supposed 408 to be part of the solution to lack of invariance, why is it not seen for these phonemes? 


\section{B. Discrimination is not categorical...}

411 A more direct challenge to CP comes from work explicitly assessing discrimination tasks. The 412 classic work on $\mathrm{CP}$ relies on the previously described $\mathrm{ABX}$ task. Ostensibly, this task does not 413 require labeling. However, it has a high memory load - listeners must encode both the $A$ and $B$ 414 stimulus and retain them, to compare them to the $\mathrm{X}$ stimulus. Here, a phonological code may be 415 a more efficient and durable form of encoding than an auditory one. That is, if the auditory 416 (perceptual) code fades, all that may be left is the categorical one. Under this view, the discrimination peaks seen in standard CP experiments do not reflect categorical perception, but rather, categorical memory or categorical judgements (Pisoni, 1973).

In response, this led to detailed analyses of the discrimination tasks that were used to assess CP (Macmillan et al., 1977; Pollack \& Pisoni, 1971); these generally led to the conclusion that the $\mathrm{ABX}$ and $\mathrm{AX}$ (same/different) tasks were perhaps the least suited for assessing pre-categorical auditory encoding, as they are both memory-intensive, and have unclear response criteria (e.g., a subject could choose to respond at a phonemic or auditory

424 level). This led to the development of discrimination tasks that are less memory-intensive or 425 biased. For example, in an "oddball" task participants hear three stimuli and identify which one 426 differs. This reduces memory demands since listeners only need to remember one token at any 427 given moment. Carney et al. (1977) used this task to show substantial within-category 428 discrimination, and no discrimination peak at the boundary. Perhaps the least biased task is the 429 4AIX task (which is highly similar to Gerrit \& Schouten's 2004, 2IFC task). In this task, 430 participants hear two pairs of stimuli, one of which differs and the other is a perfect match, and 431 their job is to indicate which pair matches. Pisoni and Lazarus (1974) first demonstrated non432 categorical perception with this task (no difference between within- and between-category 433 discrimination). More impressively, Schouten, Gerrits and Van Hessen (Gerrits \& Schouten, 434 2004; Schouten et al., 2003) first conducted a series of experiments to establish nearly perfect 
CP (perfect predictability of

436 discrimination from identification) with

437 a standard $A B X$ task. When they then

438 switched to the 4AIX/2IFC task, there

439 was no evidence for CP -

440 discrimination was not predicted by

441 identification. Thus, even playing by

442 the rules of $\mathrm{CP}$, there is no evidence

443 for a warping of discrimination when

444

445

446

447 discrimination tasks grew, Pisoni

448

449

450

451

452

453

454

455

456

457

458

459

460 we properly understand the demands

of the discrimination tasks.

As our understanding of

developed an insightful analysis that observe CP.

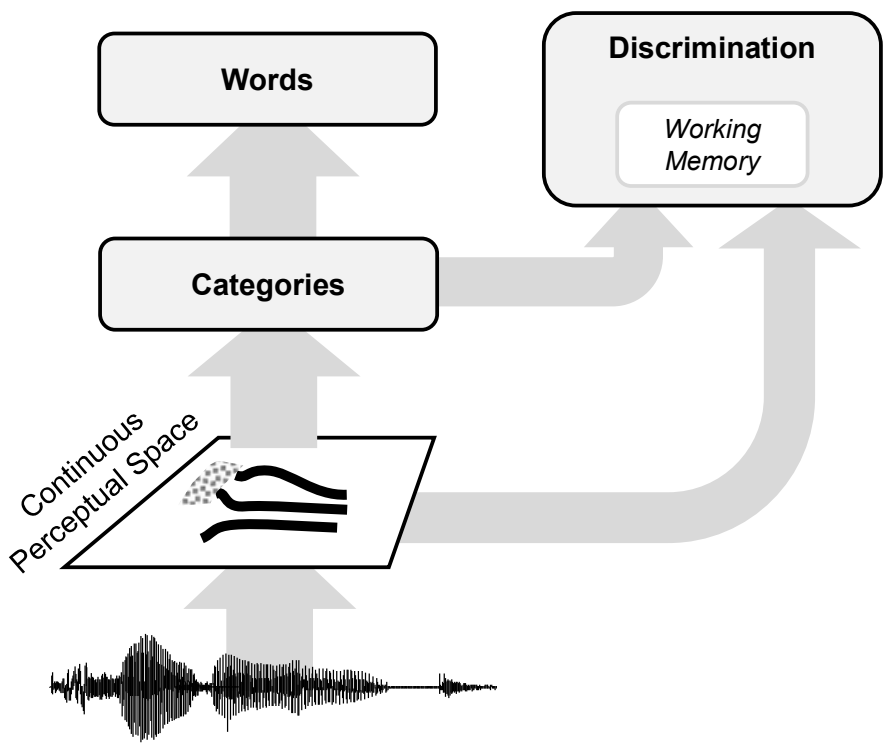

Figure 5: The Pisoni and Tash (1974) model of discrimination. Speech is transduced to a continuous perceptual space, then to categories and words (left branch). Discrimination, however, can use either the perceptual or category representation (right branch). Under these assumptions, between-category contrasts should almost always be more discriminable than within (embedded table), even if the perceptual encoding is continuous.

undercuts the premise of using any discrimination task to infer CP (Pisoni, 1973; Pisoni \& Tash, 1974). Again, he starts from a conceptualization of speech perception as a series of transformations from the continuous perceptual signal to categories like phonemes or features (Figure 5, left pathway). The typical assumption of CP is that discrimination tasks primarily tap the continuous perceptual space. However, what if discrimination judgements involve both perceptual and category information (Figure 5, right pathway)? Under this assumption, a withincategory contrast has essentially one source of evidence favoring discrimination (any auditory difference), while a between-category contrast has two (the auditory difference + the difference in labeling) (Figure 5, embedded table). That is, even if the internal perceptual difference is equivalent for within- and between-category contrasts (no sensory warping), one should still 
even if auditory perception is continuous! Any differences among discrimination tasks in their

462 ability to show CP or non-CP may derive from differences in the degree to which a given task

463 (or stimulus) differentially emphasizes category-level or perceptual-level information when

464 making the judgment. But CP can be seen even in the absence of a warped auditory encoding.

465 This is a powerful argument-largely ignored by much of the literature-suggesting a

466 fundamental limit in the degree to which the combination of identification and discrimination can

467 tell us anything about CP as a theory of speech (see also Massaro \& Hary, 1984). which discrimination tasks show CP-like performance; but b) some discrimination tasks (ABX and $\mathrm{AX}$ ) do not accurately isolate pre-categorical perceptual encoding; d) in many cases, a CP. like profile of discrimination can be predicted even in the absence of true categorical perception;

472 and consequently. As a result of this there, turns out to be little clear unambiguous positive 473 evidence for CP from the very tasks that define it. This does not rule out CP, but it leaves us 474 with little evidence for it. This prompts the need for methods to better isolate auditory encoding.

\section{C. $\quad$...and perception is continuous (mostly)}

477 Pisoni and Tash (1974) argue that discrimination tasks may not reveal the continuous or categorical nature of the auditory-perceptual space. To do this we must assess the perceptual

479 space more directly. Massaro and Cohen (1983) proposed an innovative way to do this.

480 Subjects used a continuous rating scale to rate how /b/-like or /p/-like the stimulus was (they 481 also tested place of articulation and vowels). Unlike a traditional forced choice labeling task, this 482 task can potentially reveal the degree to which listeners track the continuous input. When 483 averaged across trials, such measures were predicted to show the standard sigmoidal function; 484 however, Massaro and Cohen argued that a sigmoidal function could derive from one of two 485 underlying models (Figure 6) that can be revealed by examining the distribution of responses. 
between intermediate continuum

488 steps. Rather, they should

489 consistently choose a low rating for

$490 \mathrm{~J} / \mathrm{b} /$ and a high rating for $/ \mathrm{p} /$ (for

491 example), since they do not hear

492 differences in the middle of the

493 across continuum steps, the relative the 2AFC task).

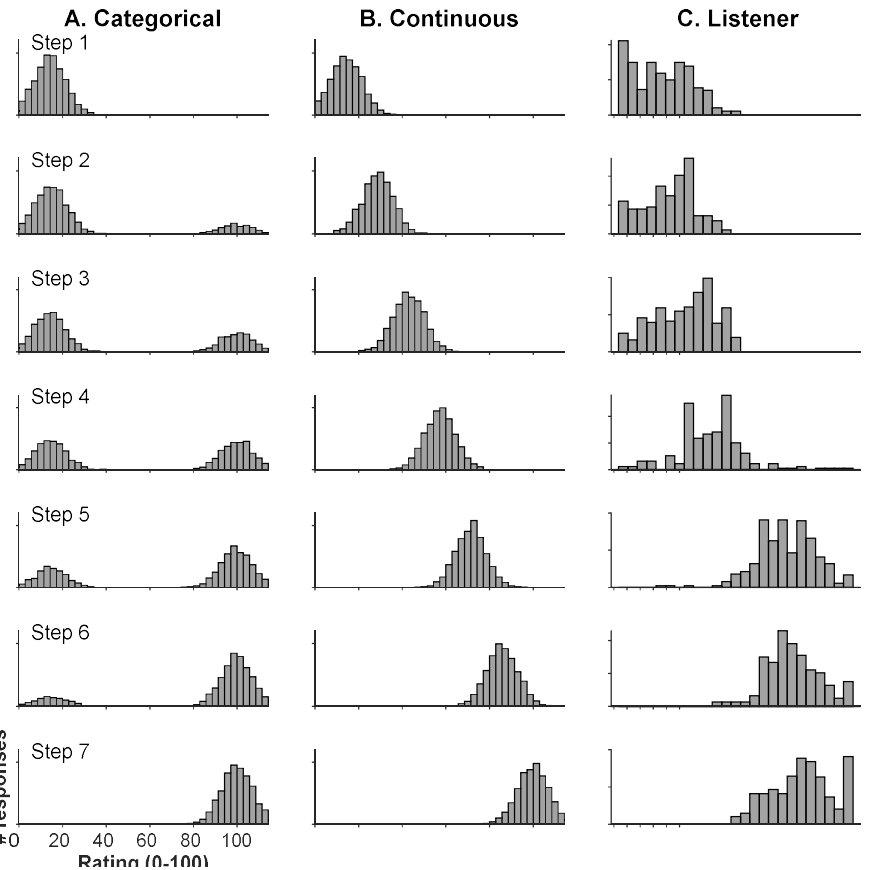

Figure 6: Distribution of rating scale responses for individual subjects as a function of continuum step (each row) from Massaro and Cohen (1983) (estimated from their Figure 7). A) Under a categorical model, listeners should use only the ends of the scale, and should only vary in how frequently they choose the $/ \mathrm{b} /$ or $/ \mathrm{p} /$ ends. B) Under a continuous model, the mean rating should shift with step. C) Estimated data from a typical listener. likelihood of hearing one or the other may vary. In contrast, if listeners encode the continuous difference between tokens, one might expect the mean rating to advance linearly as the step increases (Figure 6B). Massaro and Cohen (1983) compared the fit of these two models to individual subjects and found the continuous model offered a better fit for most subjects. This provides strong evidence of a continuous underlying auditory percept- if it was warped, listeners would not have access to the continuous information for their ratings (for replications, see Kapnoula et al., 2021; Kapnoula \& McMurray, 2022; Kapnoula et al., 2017; Kong \& Edwards, 2011) (and see Apfelbaum et al., submitted, for further discussion of this task and the limits of

More recent work has used electrophysiology to more directly assess early auditory encoding. Building on prior work by Frye et al. (2007), Toscano et al. (2010) measured event related potentials (ERPs) from the scalp while listeners categorized tokens from a VOT 

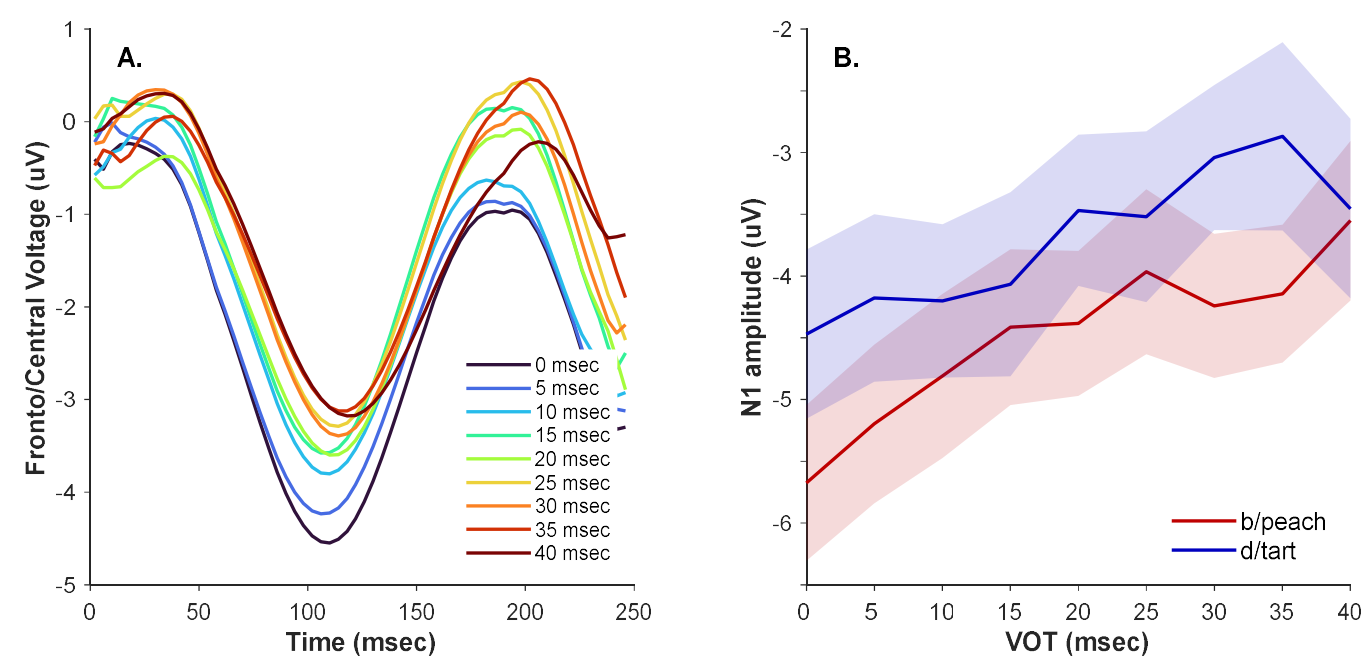

Figure 7: Results from (Toscano et al., 2010). A) Voltage at front-central channels as a function of time and VOT. The large negative deflection at $100 \mathrm{msec}$ is the N1 and its depth is related to VOT. B) Mean N1 amplitude as a function of VOT.

continuum. They examined the N1, an early negative deflection of the waveform at around 100$200 \mathrm{msec}$ that has been linked to some of the first cortical processing of sound. They found a linear relationship between the VOT of the stimulus and the amplitude of the N1 (Figure 7): shorter VOTs showed a more negative N1, which increased linearly with VOT. Critically, there was no effect of the subject's response, and no evidence for warping near the category boundary (see Sarrett et al., 2020; Toscano et al., 2018, for a replication) (Getz \& Toscano, 2021, for a review). This offers clear evidence that at the earliest stages of perception (100 msec in), the encoding of speech is not warped by the presence of categories.

Given these results, what is to be made about prior neuroscience results suggesting warping? For example, direct recordings from the human auditory cortex (Steinschneider et al., 1999) show a discontinuity such that lower VOTs have a single negative deflection, while longer VOTs have two. Such a discontinuity appears to accord with some kind of warping. However, for long VOTs, these two deflections linearly tracked the VOT - suggesting gradiency-and it is not clear whether the measurement techniques have the fidelity to distinguish two deflections at the lower VOTs where there the two peaks may be close enough to smear together. 
529 heard a place of articulation continuum, and used a representational similarity analysis to 530 demonstrate that tokens within a category were closer than those spanning a boundary (the 531 classic definition of $\mathrm{CP}$ ). However, that cortical region is also a locus of phonological processing 532 (Mesgarani et al., 2014), raising the possibility that this measure does not isolate auditory 533 encoding, but rather it may also reflect category representations. Such a model would be 534 consistent with the Pisoni and Tash (1974) model in which there is a continuous perceptual 535 encoding alongside a category representation and both contribute to disrciination (Figure 5$)^{2}$.

$536 \quad$ Finally, an intriguing challenge to linear cue encoding comes from work by Kapnoula and 537 McMurray (2022). They also applied the N1 paradigm of Toscano et al. (2010), but examined 538 individual differences by relating the N1 response function to the degree of gradiency shown in 539 a VAS task (e.g., Figure 4). They found that more categorical listeners showed a small bump at 540 the category boundary; while gradient listeners were linear. However, even the categorical 541 listeners still encoded continuous detail within each category. That is, there was a strong linear 542 trend, with an additional change near the boundary. Thus, the presumed acoustic space of this 543 subset of listeners would look more like the visualization in Figure 4C in which the distance 544 between categories is expanded but without altering the ability to represent within-category 545 detail. Such models - which may only apply in some listeners - are not commonly considered in 546 the CP pantheon, thought they are potentially consistent with it. In principle, if the N1 is picking 547 up phonological representations from STG, this may be consistent with the Pisoni and Tash 548 (1974) model (linear cue encoding + categories). However, no existing models are equipped to 549 account for such differences at an individual level, raising the need for further investigation.

\section{D. ... And meanwhile in other subdomains.}

552 Outside of the small community of researchers actively working on CP, CP appears (at least to

\footnotetext{
2 Notably, Pasley et al (2012) used similar recordings from STG to reconstruct the spectro/temporal form of heard speech. This would not have been possible with the information loss implied by CP.
} 
me) to offer a less than compelling model, and one that is often bypassed to do useful work.

For example, sociolinguistic variation (gender, dialect and identity marking) often relies on within-category variation to signal identity. If auditory representations were warped to minimize such variation, how could this important function of speech be communicated, and how could children learn to produce these markers? Similarly, speech pathologists, second language teachers and dialect coaches, must often hear fine-grained differences within a category to help clients achieve more canonical productions. Again, CP would seem to work against this important function of speech perception. I return to some of these points in the next section.

\section{E. Summary}

The foregoing discussion challenges $\mathrm{CP}$ as an empirical phenomenon at every level. CP does not appear uniformly across speech contrasts, challenging its generality as a model of perception and raising questions about which is the more common pattern. The discrimination tasks commonly used to establish CP have deep issues of memory and bias, and when these are eliminated, perception is not categorical. Moreover, the Pisoni and Tash (1974) model (Figure 5) convincingly argues that if we eliminate the assumption that discrimination only assesses the perceptual space, then CP can be observed even if that space is non-categorical (linear). The underlying representation of the continuous space - revealed by both continuous ratings and by neurophysiological measures - appears linear. And auditory perception must do more than simply yield categories, and long-recognized listening skills like talker or dialect recognition attest to the fact that some within category is preserved, and may be more difficult if we assume CP. There's no reason to hold on to CP anymore and by abandoning it, we can achieve a much more coherent and compelling theoretical account of speech recognition. 
579 phonemes, words, intention, emotion and social identity-from a continuous and variable

580 system. CP makes claims that both the level auditory representations and the level of

581 categories are quasi-discrete. At the time, this aligned with broader thinking in how the problem

582 of speech perception is solved. However, at both levels, new (and old) thinking suggests that

583 the solution implied by CP is not how listeners approach speech perception. This evidence is

584 built on the idea that speech categorization is a gradient process. As I describe, this claim, in

585 and of itself, does not constitute strong evidence against weaker forms of CP. However, it does

586 challenge the broader view of speech implied by CP.

587

588 A. Speech Categories are Gradient

589 CP implies that the goal of the perceptual system is to transduce a continuous signal to

590 something resembling a discrete category. However, substantial empirical work suggests that

591 the end result is not discrete, but gradient, and that this may be important.

592

The earliest work on this comes from Joanne Miller and colleagues (Miller \& Volaitis,

593 1989) (Miller, 1997, for a review). They used a task in which listeners heard tokens from a

594 speech continuum with a large range and rated how good of a /p/ each exemplar was. With the

595 large range, some tokens would be a /b/ and rated low, others were near typical values for a /p/,

596 and yet others were quite extreme, but not consistent with another speech sound (e.g., a VOT

597 of $150 \mathrm{msec}$ ). This allowed a visualization of the "structure" of the entire category. Critically, this

598 revealed that speech categories have a highly gradient, prototype-like structure and this entire

599 structure - not just the boundary-was sensitive to contextual factors like speaking rate (Miller \&

600 Volaitis, 1989) (see Figure 8A). Moreover, the structure and shape of these goodness ratings

601 roughly align with the distributions of VOTs that listeners experience (Figure 8B)-listeners

602 categorize speech in a way that is sensitive to the statistical distributions of those cues.

603 It is possible that early representations of phonological categories are gradient but are

604 transformed into something more discrete before accessing higher-level units like words. This 
605

606

607

608

609

610

611

612

613

614

615

616

617

618

619

620

621

622

623

624

625

626

627 was disconfirmed by later work by

Blumstein and colleagues (Andruski et al.,

1994; Utman et al., 2000). They used a

cross-modal priming task to ask if small

within-category differences in a target word

(e.g., king with VOTs that were either

prototypical or near the boundary) altered

priming for a related word (e.g., queen).

They found that even a small change in the

VOT—which do not alter the perceived

category-reduced priming, suggesting

that gradiency in speech categories is

preserved through the level of words.
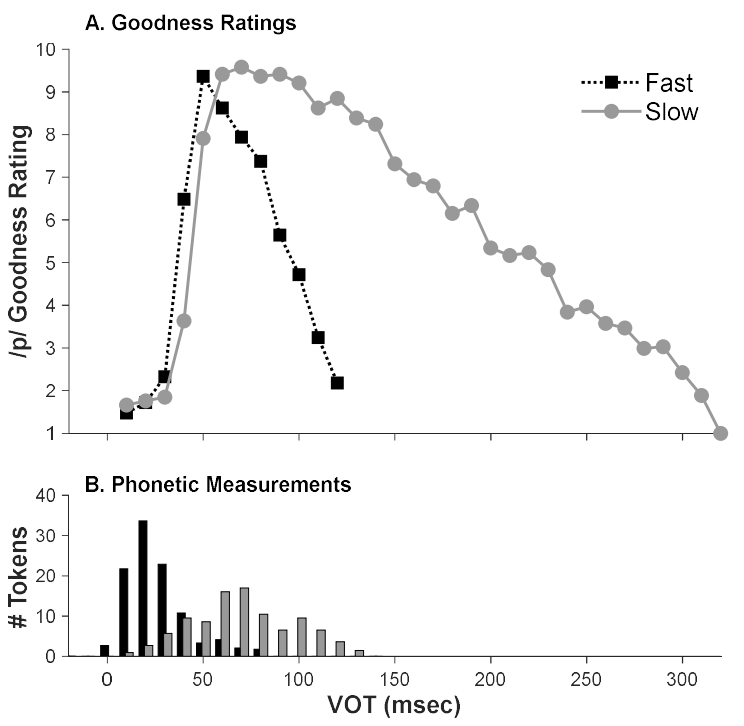

Figure 8: Typical results of phoneme goodness rating experiments of Miller and colleagues (data estimated from Miller \& Volaitis, 1989). A) /p/ goodness as a function of VOT and speaking rate (the length of the following vowel). B) Phonetic measurements showing the distribution of VOT as a function of speaking rate.

One concern with both the priming and goodness rating techniques is that they could represent an average of different types of trials. For example, in the Andruski et al. (1994) paradigm, on some trials, king may be fully active and shows full priming of queen, but on other trials it may be inactive (the listener thought they heard ging) and shows no priming. Critically, more of the trials when the VOT was close to the category boundary should fall into this latter group (in which king is inactive). This could attenuate the priming effect on the average, even if the priming effect on any individual trial was the same across VOTs (an averaging artifact). This would be consistent with $\mathrm{CP}$, but with noise from trial to trial. To rule out this concern, one needs a technique that queries which category the listener heard on that trial, while simultaneously assessing the degree of underlying activation for that category.

A number of studies have accomplished this using the Visual World Paradigm (VWP) (McMurray, Aslin, et al., 2008; McMurray et al., 2002). In these studies, listeners hear a token from a speech continuum spanning two words (e.g., beach and peach), and click on the 
631 corresponding picture. Critically, on each trial, the words and their locations change,

632 necessitating one or more eye-movements to plan the motor response. These fixations can

633 reveal the underlying activation of a word. For example, if the listener heard beach, fixations to

634 the peach prior to the response can indicate how active this competing word is.

635 These studies have taken several steps to rule out the averaging artifact. First, each

636 subject's own category boundary was computed from their identification data, and all data were

637 recoded relative to that boundary. Here, $-5 \mathrm{msec}$ of VOT then refers to $5 \mathrm{msec}$ from the

638 boundary (toward $/ \mathrm{b} /$ ), and +10 is $10 \mathrm{msec}$ toward $/ \mathrm{p} /$. This avoids the possibility that increased

639 looking is driven by variation in the boundary across subjects. Second, the analysis of fixations

640 is conditioned on the response by discarding trials in which the "incorrect" response was

641 chosen. That is, given that the listener heard a sound on the /b/ side of their boundary, and that

642 they ultimately reported a /b/, do competitor fixations nonetheless vary as a function of VOT?

643

With this analytic approach, these studies typically find a linear effect of VOT on

644 competitor fixations. Figure 9A shows looks to the competitor (/p/ when hearing a token on the

$645 \mathrm{lb} /$ side of the continuum) as a function of time and relative VOT (from McMurray et al., 2002).

646 As the VOT moves toward the boundary (at relative VOT=0), there are systematically more

647 fixations to the competitor. Figure 9B shows the area under the curve and shows the same

648 gradiency as a function of VOT (for replications, see Kapnoula \& McMurray, 2022; McMurray,

649 Aslin, et al., 2008; McMurray et al., 2014). This analytic approach has also been applied to an

650 ERP index of categorization, the P3 (Kapnoula \& McMurray, 2022; Toscano et al., 2010). Here,

651 a stronger P3 indicates more robust categorization. As with the VWP studies, these analyses

652 accounted for both the subject's boundary and their response on each trial and find a stronger

653 P3 for more prototypical VOTs. Critically, these findings are highly robust across individuals.

654 Kapnoula and McMurray (2022) tracked individual differences in the gradiency of a listener's

655 speech categorization using the continuous VAS task and related it to both VWP and ERP/P3

656 measures. They found correlations between these measures: more gradient VAS responding 

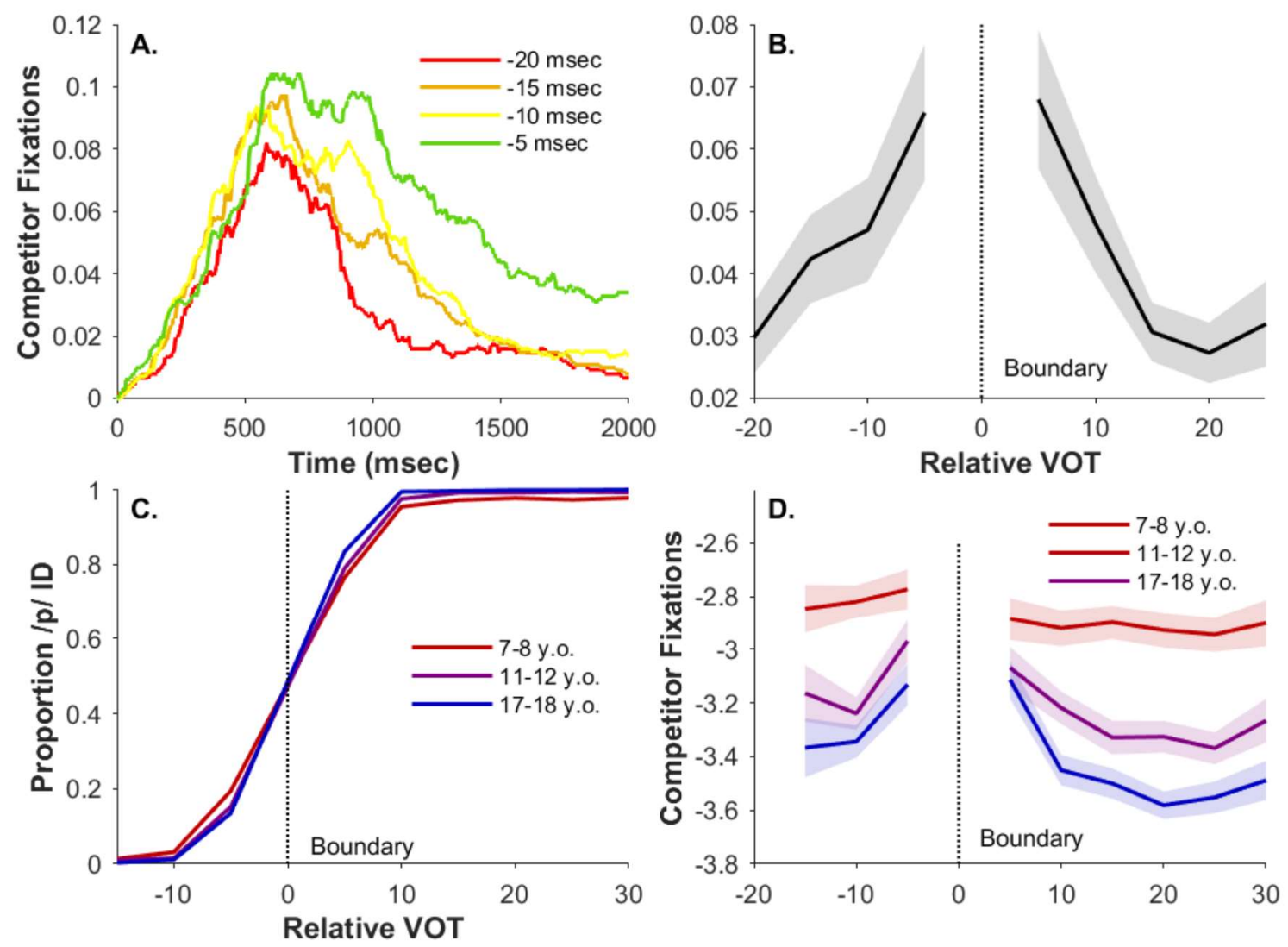

Figure 9: Results of VWP experiments documenting gradient within-category sensitivity to finegrained detail. A) Fixations to the competitor as a function of time, and distance from the category for tokens on the voiced side of the continuum from McMurray et al. (2002). B) Average fixation from 200 to $2200 \mathrm{msec}$ as a function of relative VOT in that study. C) 2AFC labeling function for children in McMurray et al. (2018); here, identification becomes steeper (more categorical) with development. D) In contrast, competitor fixations as a function of relative VOT show increasing sensitivity with development.

led to more gradient competitor fixations and P3s. However, critically, all listeners were gradient

658 (see also Kapnoula et al., 2021), they just varied in degree.

The results from goodness ratings, the VWP and ERPs all suggest that speech categories are highly gradient-even when we control for the labeling on each trial and for variance in participants' boundaries. This provides indirect evidence against an entirely discrete representation at the auditory/perceptual level: if these representations were discrete, withincategory detail would not be available to higher-level phoneme or lexical processes. This is not 
665

666

667

668

669

670

671

672

673

674

675

676

677

678

679

680

681

682

683

684

685

686

687

688

reduced, but not lost, and yet is sufficient to support a gradient representation. The point is not that these kinds of results directly refute CP. Rather, gradiency conflicts with the more discrete results, commonly seen in $2 \mathrm{AFC}$ identification tasks, and it challenges the functional "goal" of speech categorization implied by CP. That is, the goal of speech perception is not to ignore irrelevant variation within a category but to systematically track it. Two extensions of this work highlight this fact in distinct ways.

First, this mismatch between the underlying [gradient] activation and the overt [categorical] labeling is highlighted by a recent developmental study. McMurray et al. (2018) tested children from 7 to 18 in a variant of the McMurray et al. (2002) paradigm. When we examined the identification curves (Figure 9C), we found increasingly steep categorization with development. This would appear to be consistent with a view in which children become increasingly categorical over development. By the standard view of $\mathrm{CP}$, this implies they increasingly lose access to fine-grained detail. However, the fixations showed the opposite pattern (Figure 9D): the 7-8 year-olds (red curve) showed essentially no sensitivity to finegrained detail, and this emerged in later years. Thus, rather than development leading to the loss of sensitivity, it appears that children achieve greater sensitivity to fine-grained detail with age, and this in turn enables apparently sharper categorization ${ }^{3}$. This undercuts a core assumption of CP but also suggests limits to 2AFC categorization (Apfelbaum et al., submitted). Second, Myers et al. (2009) used fMRI to localize the neural basis of speech categorization. They found a highly gradient pattern in Superior Temporal Gyrus (STG)—an auditory/phonological area, but a more categorical response function in the inferior frontal gyrus, a down-stream language area (see also Toscano et al., 2018). This supports a model closer to that of Figure 5 in which the system maintains both a continuous perceptual representation of the signal (in earlier areas like STG), and something more abstract and cognitive (in IFG).

\footnotetext{
3 This mirrors work by Van Hessen and Schouten (1999) which found that increasing the quality of the continuum—enhancing access to fine grained detail-led to sharper categorization and more robust CP.
} 


\section{B. ....and Gradiency is Helpful}

691 Modern theories of speech perception all agree that auditory input is represented continuously,

692 and that activation for categories is gradient. This is true for a wide variety of theories from

693 radically different theoretical groundings, including theories from an information integration

694 perspective (McMurray \& Jongman, 2011; Nearey, 1997; Oden, 1978), connectionism

695 (McClelland \& Elman, 1986), Bayesian/ideal observer frameworks (Kleinschmidt \& Jaeger, 696 2015), auditory accounts (Kluender et al., 2003), and exemplar theories (Goldinger, 1998). This

697 is for a good reason - many of these theories posit representations or operations that are simply

698 incompatible with a discontinuous auditory input or a discrete category. CP is fundamentally

699 about losing or reducing access to (within-category) information in the signal, while all of these

700 theories attempt to maximize how this information is maintained and used.

701

It is beyond the scope of this paper to attempt a detailed review of all of these theories.

702 However, it is important to discuss the general principles, the facts about speech, and the

703 empirical results that argue that gradient representations at every level are beneficial for solving

704 the challenging computational problem of speech perception. In this section, six perceptual

705 problems illustrate the inconsistency between CP as a theoretical account, and the way most

706 people now think about speech, and they augment arguments for the gradient alternative.

\section{1. Cue Integration.}

708 It has long been known that multiple cues contribute to phonemic percepts (Lisker, 1986; Oden

709 \& Massaro, 1978; Repp, 1982). For example, voicing is primarily cued by VOT (in English), but

710 factors like F0, F1 frequency, the length of the subsequent vowel and the preceding speaking

711 rate all contribute. These sort of trading relationships often appear as a shift in the boundary

712 along a primary dimension (e.g., VOT) as a function of a secondary cue (Figure 10A). Critically,

713 to perform these kinds of sensitive boundary shifts, listeners must be able to encode cues with a

714 fairly high degree of precision. If all they heard was $/ \mathrm{b} /$ or $/ \mathrm{p} /$, it is not clear how this could occur. 

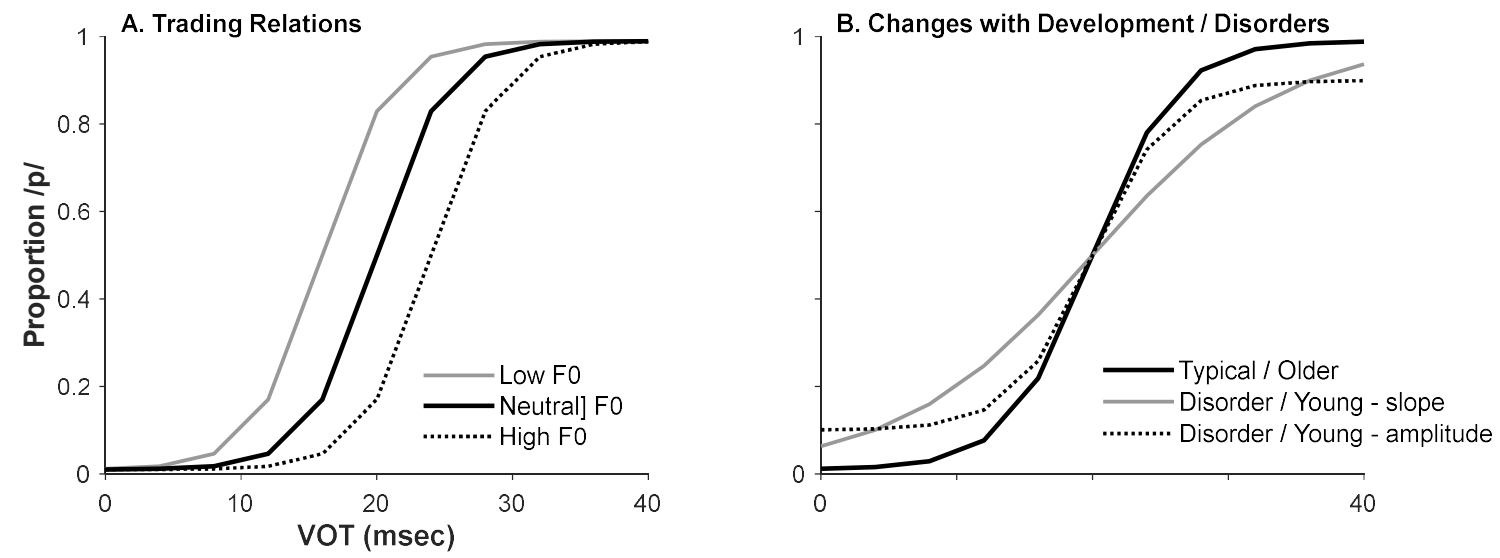

Figure 10: A) Schematic results of a typical trading relations experiment examining voicing categorization (/b/ vs. /p/) as a function of VOT (x-axis) and F0 (separate curves). B) Schematic results of an experiment examining voicing categorization in younger and older children, or in disordered and typical children.

This argument has been used frequently - including by me (Kapnoula et al., 2017).

However, the fundamental work on trading relations was not seen as inconsistent with CP

717 (Repp, 1982). Part of the issue is the question of what is warped by CP: an individual cue (like

718 VOT), or a multi-dimensional space. If cues are coded independently (and warped

719 independently) this argument holds - if VOT is collapsed into /b/ or /p/, it cannot be integrated

720 with F1 or F0 in any meaningful way because the fine-grained differences are lost. However,

721 what if phonological dimensions are a composite across cues (e.g., a combination of VOT and

722 F0), as a sort of two-dimensional space with a diagonal boundary (e.g., Figure 4A). Under this

723 assumption, trading relations may come for free without the need for actively shifting the

724 boundary. For example, in Figure 4A we could imagine a continuum of spectral mean (along the

725 horizontal dimension) with an F2 onset of $1500 \mathrm{~Hz}$. Here, the boundary should be around 5000

$726 \mathrm{~Hz}$; in contrast, with an F2 onset of $2500 \mathrm{~Hz}$, the boundary needs to be at $6000 \mathrm{~Hz}$. However, if

727 we assume that the auditory representation is multi-dimensional, the spectral mean boundaries

728 does not shift due to a secondary cue (F2); rather the multi-dimensional space is simply

729 sampled along a different orientation (the diagonal). Critically, CP could operate over the entire

730 space (Figure 4B), not on individual cues, and thus could be consistent with trading relations.

731 While this appears to reconcile CP and trading relations, it may be insufficient for two reasons. 
First, for most speech sounds, not all cues arrive at the same time. In word-initial voicing, vowel length arrives after VOT; for sibilant fricatives, formant frequencies arrive after the frication spectrum. A wealth of work suggests that listeners do not wait to integrate cues, but rather use each cue as it arrives (McMurray, Clayards, et al., 2008; Reinisch \& Sjerps, 2013; Toscano \& McMurray, 2015) (though see Galle et al., 2019). This means that even if the auditory space is multi-dimensional, at least for a brief period, some cues need to be coded in isolation. If CP operated then on these individual cues, it would be hard to integrate later cues when they arrived. Consequently, a gradient representation may be needed to avoid making an erroneous commitment on the basis of an initial cue while waiting for later cues.

Second, a number of studies have used an individual differences paradigm to assess this (Kapnoula et al., 2021; Kapnoula et al., 2017; Kong \& Edwards, 2011; Ou et al., 2021). These experiments use a continuous rating scale similar to Massaro and Cohen (1983), now referred to as a Visual Analogue Scale (VAS) task (see Apfelbaum et al., submitted). Subjects' gradiency is quantified as a continuous estimate of their slope and this can be related to other measures. On balance, these studies show evidence for the hypothesis that gradient speech categories are functionally beneficial. Gradiency in VOT has been linked to the degree to which listeners also use F0 (Kapnoula et al., 2021; Kapnoula et al., 2017; Kong \& Edwards, 2011; Ou et al., 2021); and gradiency in tracking formant frequencies has been correlated with the use of duration in vowel judgements (Ou et al., 2021); however, correlations have not been observed for other cue combinations such as fricative spectra and formant transitions, or VOT and vowel length (Kapnoula et al., 2021). This may derive from issues such as when the cues arrive (relative to each other), or whether cues are coded independently or in some kind of multidimensional space (see also Toscano \& McMurray, 2010). However, for at least some cues, the claim that a gradient representation is linked to better cue integration is observed empirically.

\section{Accounting for Context.}

A related phenomenon is context. Phonetic boundaries also shift as a function of factors that 
758

759

760

761

762

763

764

765

766

767

768

769

770

771

772

773

774

775

776

777

778

779

780

781

782

783

themselves do not directly cue a phoneme. For example, listeners can shift fricative boundaries depending on the talker (Strand, 1999) or neighboring sounds (Mann \& Repp, 1981). This is distinct from trading relations. Whether or not a speech sound was spoken by a male or female does not directly inform the listener whether a sound is /s/ or $/ \int /$. However, both physiological and sociolinguistic differences lead male and female talkers to produce these sounds differently. Consequently, listeners can do better if they can adjust their boundaries based on the context. As with cue integration, such operations require a fine-grained analysis of the signal, and they may require retaining early phonetic cues until later context arises - for example, in a fricativevowel sequence, the talker identity may not be available until the onset of the vowel.

Work on the C-CuRE (Computing Cues Relative to Expectations) model of speech perception exemplifies the need for a fine-grained representation of the continuous cues to deal with context (Cole et al., 2010; McMurray \& Jongman, 2011). In this model, phonetic cues are encoded continuously, but they are adjusted relative to contextual expectations. For example, an $\mathrm{F} 0$ of $160 \mathrm{~Hz}$ is recoded as $40 \mathrm{~Hz}$ below the expected pitch for a woman $(200 \mathrm{~Hz})$. Critically, when this simple strategy is applied to real phonetic measurements, it has been shown to account for virtually all the variance in F1/F2 measurements in a corpus of vowels featuring 10 talkers and multiple coarticulatory contexts. Moreover, McMurray and Jongman (2011) applied this to a large corpus of fricatives featuring all 8 fricatives, 20 talkers, and multiple vowel contexts (Jongman et al., 2000). They showed that a simple classifier model based on these adjusted cue-values could yield a pattern of performance that closely mirrored that of listeners (both in accuracy and the pattern of errors), while the same model trained on raw cues was both substantially less accurate and did not match listeners' patterns of errors. Figure 4D offers a visualization of the same fricative cues from $4 \mathrm{~A}$ but after compensating for talker and vowel differences. Note here, that like the CP models, variance is reduced. But unlike those models, this variance is not just discarded; it was used to make inferences about the talker and the vowel. Moreover, discriminability is now increased. The discriminant analysis went from $92 \%$ to 
$96.6 \%$ correct, while preserving the continuous variability. Thus, it is possible to obtain the supposed benefits of CP without throwing away within-category detail (in fact, by harnessing it).

This work speaks to the value of a continuous representation of acoustic cues (contra CP). Given that any cue may be adjusted on the basis of multiple factors (talker, coarticulation), and by small amounts, the system must retain as close to a continuous representation as possible to get the most out of this form of processing. Critically, like CP this model does not posit a veridical representation of the cue: cue values are coded relative to internally generated expectations (e.g., this spectral mean is low for a woman). However, the fundamental operations are different: CP dichotomizes a dimension in a way that rather arbitrarily eliminates variability, whereas C-CuRE shifts the estimated cue value to more sensitively account for context. Again, these kinds of processes are not inconsistent consistent with a partial form of $\mathrm{CP}$ in which within category differences are reduced but not lost. But at the same time the nonlinearity imposed by the categories on the signal may add substantial noise, making this compensation accurate. Moreover, the nature of the compensation is at odds (harnessing variance) with the CP-inspired theoretical view (of ignoring it). Given this, what is the value of hanging on to $\mathrm{CP}$ in some kind of reduced form?

\section{Listeners do more than one thing with any speech cue.}

Perhaps the deepest challenge to CP is rooted in an observation made by Mermelstein (1978): every speech cue is used for more than one thing (see also, Smits, 2001; Whalen, 1992). For example, in English, vowel length may serve as a cue to speaking rate, it may distinguish tense and lax vowels, and it contributes to both syllable-initial and syllable -final voicing! This was a fundamental part of the indeterminacy that comprises the problem of lack of invariance: each cue value can be affected by multiple factors, and each factor affects multiple cue values.

The problem is that if a listener categorically warps the auditory input for one set of categories, he or she may lose information that could be used for another. For example, the spectral mean of a fricative is a robust cue for whether it is $/ \mathrm{s} / \mathrm{or} / \mathrm{J} /$. However, that same cue 
810 also contains useful information for the upcoming vowel: /s/ before a rounded vowel like /u/ will

811 have a lower spectral mean than before an unrounded vowel like /i/ (Daniloff \& Moll, 1968).

812 Critically, both /si/ and the /su/ have spectral means that are still within the boundary for /s/.

813 Consequently, if CP operated to minimize within-category differences, these subtle differences

814 would be lost and listeners would not be able to use the frication to anticipate an upcoming

815 vowel-as they clearly can do (McMurray \& Jongman, 2015; Yeni-Komshian \& Soli, 1981).

816 Virtually every speech cue is affected by multiple factors (a key underpinning of the

817 problem of lack of invariance). CP ignores this, to focus on the coding and perception of a

818 limited number of cues for a single phonetic contrast. But when we consider CP in light of this

819 basic fact about speech perception, it is hard to see how perception can be discontinuous, and

820 yet listeners could still use all of this gradient information.

\section{4. Speech Cues do not just encode Phonological Categories.}

822 Building on this, speech cues do more than just carry categories. VOT is related to the gender

823 of the talker (Allen et al., 2003), fricative spectra carry information about gender (Zimman, 2017)

824 and the degree to which the talker identifies as part of the gay community (Munson, 2007); and

825 of course vowels carry substantial information about dialect and language community. Beyond

826 social marking, cues like F0 which are used to signal vocal emotion also contribute to vowel and

827 consonant identity (Ohde, 1984). And people must use fine-grained within-category differences

828 to tune both their own productions (e.g., to cope with something in their mouth or a missing

829 tooth), or others (e.g., a parent or speech pathologist working with a child). To the extent that

830 within-category information is entirely lost (the strong form of $\mathrm{CP}$ ), these judgements become

831 even impossible. But even under weaker forms of CP they become more difficult. In this view,

832 CP appears to privilege the goal of identifying phonological categories over these other crucial

833 roles of speech. In contrast, a continuous representation of the auditory space coupled to

834 gradient categories would not sacrifice these abilities. While these fields have not widely

835 wrestled with the deep implications of these functions for CP (I could find few published papers), 
836

837

these phenomena are widely acknowledged, and my view as an outsider is that fields like sociolinguistics or speech pathology have often just ignored CP and moved on (though see Plichta \& Preston, 2005, for an elegant demonstraton of non-categorical perception of geographic dialect). However, abandoning CP as a core property of speech, may permit more unification of our models of phoneme recognition and these other functions.

\section{Flexibility.}

A gradient representation - at the level of categories - has also been linked to perceptual flexibility. That is, even with multiple cues and compensation mechanisms, the speech input may be ambiguous due to speech errors, an unfamiliar accent or context, or just plain variability. In these cases, it may be helpful to avoid making a strong categorical commitment and keep options available in case later information suggests the need to revise this initial commitment. McMurray et al. (2009) offered one of the first tests of this using a "lexical garden path" paradigm inspired by work in sentence processing. Participants responded to words from a $\mathrm{p} / \mathrm{b}$ continuum that created a form of temporary ambiguity. For instance, in a word like parricade, if the onset sound was ambiguous between $/ \mathrm{b} /$ and $/ \mathrm{p} /$, the word could be briefly consistent with both barricade and parakeet, and resolution would not occur until late in the word (at -cade or keet). This study tested listeners' ability to recover from these ambiguities in a VWP experiment. When listeners heard parricade with a VOT of $40 \mathrm{msec}$, they were initially biased to interpret the input as the beginning of parakeet, and then would need to revise their decision when -cade arrives. However, when the VOT was $30 \mathrm{msec}$ (still a /p/), listeners were still biased to parakeet, but recovery was faster, because barricade was more active. In contrast, if listeners had been categorical, there would not be an ongoing activation of barricade for either VOT, as the activation of $/ \mathrm{p} /$ necessitates full suppression of $/ \mathrm{b} /$. Under $\mathrm{CP}$, then, they could not have shown this gradient recovery. Several additional VWP experiments show supporting evidence suggesting that a partial commitment is helpful for maintaining this flexibility (Brown-Schmidt \& Toscano, 2017; Gwilliams et al., 2018; Szostak \& Pitt, 2013). Critically, Kapnoula et al. (2021) 
862 tested subjects both in this lexical garden-path paradigm and in a VAS / speech categorization

863 task (as in Figure 6). As in her prior work, Kapnoula and colleagues used the VAS to establish

864 the relative amount of gradiency and found that participants with a more gradient profile of

865 speech categorization were better able to recover from the garden-path. This validates a core

866 prediction of this argument—more gradiency leads to more flexibility—while also raising

867 questions about individual differences that are not accounted for by current models.

In principle, one could argue that these sorts of effects could be handled by a system

869 that was initially gradient, but which rapidly resolved to more discrete (either/or) commitment to

870 a category (in the lexical garden-path paradigm, ambiguity was typically resolved by around 250

$871 \mathrm{msec}$ ). However, later studies using sentential context show that these benefits can last a

872 second or more (Brown-Schmidt \& Toscano, 2017; Falandays et al., 2020). Moreover, ERP

873 work by Sarrett et al. (2020) applied the N1 / VOT paradigm of Toscano et al. (2010) (Figure 5)

874 to complex sentences and found a linear effect of VOT on the EEG as late as $900 \mathrm{msec}$ after

875 the VOT. Critically, the target word was at the end of these sentences-no further information

876 was coming. Thus, participants maintain a gradient representation for quite a while, even if they

877 aren't going to need it. Again, we also point out that this kind of gradiency is not strong

878 evidence against any but the most extreme form of CPs. But again, this kind of gradient

879 sensitivity is clearly at adds with the notion of a quasi-discrete category space.

$880 \quad$ 6. Learning / Plasticity.

881 Finally, gradiency may be necessary for learning and adaptation. Both infants and adults take 882 advantage of statistical learning mechanisms to acquire the categories of their language (Maye

883 \& Gerken, 2000; Maye et al., 2008; Werker \& Curtin, 2005), acquire new categories of a second 884 language (Escudero et al., 2011; Escudero \& Williams, 2014), and tune existing categories to 885 adapt to novel talkers or contexts (Munson, 2011). These mechanisms work by tracking the 886 frequency of occurrence of individual cue-values (e.g., how frequent is a VOT of 20 or $25 \mathrm{msec}$ ); 887 they use the kinds of distributions depicted in Figure 6B to identify the number of categories 
888

along a dimension as well as their prototypical values and the allowable extent.

While there is debate about the sufficiency of these mechanisms to fully account for firstlanguage speech category acquisition (McMurray, in press; Schatz et al., 2021), it is clear that listeners can learn from such statistically structured input (Escudero et al., 2011; Maye et al., 2003). In fact, to some extent, the gradiency observed at the level of categories (e.g., Figure 8) may reflect the fact that these categories are a product of statistical learning. That makes sense if the goal is to adapt the system to the degree of uncertainty: when there is clear evidence that a given token is a /p/, there is no need to hedge one's bets, but when the current input is from a less frequent region of the space it may be useful to withhold a strong commitment (McMurray \& Farris-Trimble, 2012). This kind of statistical learning is impossible in a system that doesn't preserve speech cues in a veridical form (e.g., if we assume CP). That is, to track the frequency of specific cue values (e.g., how often a VOT of 20 msec occurs), listeners must maintain a continuous representation of the cues (McMurray \& Farris-Trimble, 2012).

Of course, standard views of infant development assume a more or less categorical result of development (Werker \& Curtin, 2005); these models might argue that babies are continuous or gradient long enough for such mechanisms to acquire the phonemes of the language, at which point a more categorical system develops. However, two arguments speak against this. First, even after infants have achieved native-language like discrimination of voicing (at 4 months), they discriminate speech in a gradient, not categorical manner (McMurray \& Aslin, 2005; Miller \& Eimas, 1996). Second, statistical learning of this sort is clearly operative in adults and may play an important role in second language acquisition or in fine-tuning categories to new dialects or talkers. A categorical representation in adults would preclude these mechanisms from being used for adaptation in adulthood.

The most compelling link between these kinds of gradient representations, plasticity and flexibility comes from a series of studies manipulating the amount of variability faced by subjects (Clayards et al., 2008; Theodore \& Monto, 2019). In these studies, listeners performed a series 
914 of standard identification or word recognition trials with stimuli spanning a speech continuum.

915 However, unlike most studies, the distribution of stimuli across the continuum was not uniform.

916 For some subjects, the distribution was tightly clustered around two prototype values (e.g.,

917 VOTs of 0 and $40 \mathrm{msec}$ ) with little variation; for others, the distribution had a much higher

918 variance, creating more uncertainty. Critically, the subjects that received wider distributions

919 showed shallower identification slopes, or more gradient eye-movement responses. Thus, fine-

920 grained continuous detail is used not only to track the distributions of cues that comprise

921 phonetic categories, but the learning itself is geared toward managing uncertainty in the system.

922

923 C. Summary

924 As this review attests, there is not strong evidence that fundamental auditory representations

925 are warped by categories (Gerrits \& Schouten, 2004; Schouten et al., 2003) and mounting

926 evidence that it is not (Toscano et al., 2018; Toscano et al., 2010). Further, speech categories

927 are gradient, and this gradiency is preserved both at later levels of the system (e.g., lexical)

928 (Andruski et al., 1994; McMurray et al., 2002) and for considerable periods of time (Falandays et

929 al., 2020). As I have described, this kind of gradiency is not inconsistent with weaker (and more

930 realistic) forms of $\mathrm{CP}$ - as long as some amount of within-category sensitivity is available,

931 listeners can exhibit a gradient category. However, that is not the point of this argument.

932 Many if not most theories of speech perception are motivated in part by design principles

933 (Kapnoula \& McMurray, 2022): processes and representations are built in part to help listeners

934 functionally overcome the difficult problem of categorizing speech (chiefly, the problem of lack of

935 invariance). A continuous representation of speech cues and a gradient representation of

936 categories offer a number of useful design principles that are embraced by widely diverging

937 theories. Gradiency may help with integrating multiple cues and contextual factors; it is

938 necessary to deal with the fact that speech cues serve multiple goals-phonetic, articulatory

939 and sociolinguistic; it helps maintain flexibility in the face of uncertainty; and it is an essential 
940

941

942

943

944

945

946

947

948

949

950

951

952

953

954

955

956

957

958

959

960

961

962

963

964

965

ingredient-and product of-perceptual plasticity and learning. The essence of CP is an arbitrary reduction of withing-category phonetic detail information; however, all of these functional benefits require the opposite: preservation or utilization of such detail (something which children are trying to achieve over development, Figure 7D). While gradiency will not solve the problems of lack of invariance alone (Kapnoula \& McMurray, 2022), the benefits of this type of processing are too wide-ranging to ignore.

\section{Can some form of CP be saved? And should we?}

At this point many readers may be looking for alternatives. Can some form of CP be salvaged? Given the state of the evidence, CP cannot be fully ruled out. But why keep it?

First, let's consider what we can conclude. Clearly, there is some within-category sensitivity, and there is some level of the system that encodes speech cues linearly. But could there be other auditory representations that are warped in some way? Perhaps.

It is difficult at this point for discrimination tasks to provide strong positive evidence for $\mathrm{CP}$ - there are too many alternative approaches (e.g., Figure 5) that have not been ruled out. But what about the gradiency at the level of categories? In fact, a gradient category representation can live on top of (or in parallel to) a partially warped can have a gradient categorization on top or in parallel to a partially warped cue encoding. So, none of the work on gradient categories completely rules out weaker forms of CP. Perhaps more hopefully (if you want to retain some version of $\mathrm{CP}$ ), the neuroscience is still poorly understood and can be interpreted in terms of some kind of hybrid. The prior work showing a partially warped N1 in some listeners (Kapnoula \& McMurray, 2022) could be an example of some form of CP (in at least some listeners), or it could be explainable in a Pisoni and Tash (1974) model as the simultaneous contribution of a linear cue encoding and a category on the N1. Similarly, the MMN (Phillips, 2001) is well-replicated as a tool for eliciting CP. It is unclear if this too reflects simultaneous comparison at cue- and category-levels; or if it is strong evidence for CP. The fact 
966

967

968

that it is pre-attentive (it can be elicited while subjects sleep), rules out explicit strategies, but not necessarily implicit category activation. Thus, we need more work on these basic neuroscience measures to understand what they are assessing.

Even as we can't rule out CP, is there really that much evidence for CP? Once you understand the discrimination tasks, it is less clear that any of these constitute strong evidence for it. And the alternative theoretical model is compelling. CP is a strong claim that something that supposedly occurs prior to categorization (auditory encoding) is warped by categorization. It requires strong evidence. The converse: Categorization acts to carve up continuous cues is not controversial. So given the dearth of hard evidence, and to the extent that many studies can be explained by simple categorization, maybe we just want to posit that categorization occurs.

Given that we know there is some level of continuous encoding, the real question isn't just is there or isn't some additional a warping somewhere else in the system. The real question is whether listeners (and downstream speech processes) have access to a veridical representation of the speech signal (and they clearly do), and which kind of representation is more important for language. Even the original conception of CP would be perfectly happy with a veridical representation that was transformed into a warped one, but that this warped one was the basis of downstream processes. Rather showing that this veridical one is available to support downstream processes or behavior is the crucial insight (and the evidence presented here clearly suggests it is). And even if you want to admit a role for some kind of warped representation of the auditory encoding, given the role of fine-grained detail in most modern theories of speech do we really want to make CP so central to our field? Should it really be the one of the few things that are taught about speech perception in introductory cognition tasks?

\section{Or is it a side issue or a caveat to the prevailing modl?}

Finally, can be argued that I'm splitting hairs. Perception isn't just auditory encoding—it should include anything that reaches the level of awareness. I agree. The arbitrary line between cognition and perception is old fashioned and out of date. But if we embrace this more holistic 
992 view, CP becomes trivial. That is, it just means that people categorize things. Part of the

993 theoretical richness of CP is that it offers clear definitions about levels of the system that allow it

994 to posit counterintuitive mechanisms. But by expanding our notion of perception to include

995 categorization, when we argue that people "perceive" items within a category as more similar,

996 that also entails the trivial point that they categorize them more similarly, or that they judge the

997 more similarly. Under this assumption, what is the added insight of invoking CP?

998

\section{CP is dangerous for other fields}

The foregoing discussion has focused on whether speech is perceived categorically or gradiently - a set of issues that are internal to CP. However, CP has also been impactful in a whole range fields other than the narrow community that has worked on this core problem in typical adults. It has informed theoretical debates external to CP on the nature of speech categories over development, in communicative disorders and bilingualism/L2 speech perception. In these domains, the question is not whether or not speech is perceived categorically; rather, studies have assumed CP to make insights into the nature of perception in different populations. That is, CP serves as a sort of linking hypothesis to allow us to make inferences about speech perception from an empirical paradigm. But with the fall of $\mathrm{CP}$, where does that leave these endeavors? In fact, CP has arguably led to incorrect conclusions in many cases. Moreover, CP has informed work outside of speech in other domains of perception. Here, the presumption of CP has supported claims about modularity or linguistic relativity, but often without an attempt to wrestle with the trickiness of discrimination tasks, or with ideas like the Pisoni and Tash (1974) model. I briefly discuss these various domains before concluding.

\section{A. Infancy.}

There has been near consensus in the developmental literature on how speech categorization develops (Kuhl, 2004; Werker \& Yeung, 2005) (but see McMurray, in press, for a more critical 
1018

1019

1020

1021

1022

1023

1024

1025

1026

1027

1028

1029

1030

1031

1032

1033

1034

1035

1036

1037

1038

1039

1040

1041

1042

1043

review). Seminal work by Eimas et al. (1971), Werker and Tees (1984) and Kuhl (1979) argues

that infants start with the ability to discriminate many (if not most) speech contrasts in the world's languages, and their perceptual abilities narrow over the first 12-18 months such that only the sounds of their language are discriminable. Given that infants at these ages know few words, cannot produce speech, and get little direct social feedback, the assumption is that this development is guided by some form of statistical learning (Maye et al., 2003). This body of work appears to challenge the account laid out here as the changes in infant discrimination appear to reflect increasing warping of speech categories over development. However, as I describe, the modern understanding detailed here offers a simpler explanation. Moreover, in a somewhat circular fashion, the canonical view of speech development (c.f., McMurray, in press) was built on two strong assumptions from CP. Consequently, the demise of CP poses fundamental challenges to it which I describe next.

\section{Does infant work support CP?}

The dominant view of infant speech development is one of progressive loss of within category sensitivity. This would seem to fit neatly with CP. However, the framing of (Pisoni \& Tash, 1974; Figure 5) offers a compelling alternative. These results are also consistent with a model in which infants have categories (or even sets of micro-categories: Schatz et al., 2021) alongside a veridical auditory/perceptual representation and both contribute to the response (much like in adult discrimination tasks). In this light, consider a standard habituation/dishabituation design in which discrimination is tested by repeating a single baseline stimulus and then changing it to either a within-category variant or a between-category variant (with the same physical distance) (e.g., Eimas et al., 1971). It is reasonable to expect a larger dishabituation response when the stimulus seems more novel. Thus, when the baseline stimulus changes from a /b/to a/p/it is more novel to the infant as it differs at both the cue and the category level, than if it changes from one /b/'s to another /b/ with a different VOTs. That is, even without a warping of the auditory encoding, the presence of some kind of category on top of that may be sufficient to 
1044 drive what appears to be increasing sensitivity to the native language. In this model, what is 1045 changing with development is not any kind of warping of the space, but rather, the strength and 1046 confidence of the category representations which gradually come to dominant the sense of 1047 novelty (discrimination). Supporting this, meta-analyses (Galle \& McMurray, 2014; Tsuji \& 1048 Cristia, 2014) and individual studies (Kuhl et al., 2006) suggest that the growth of between1049 category sensitivity may be a dominant pattern of change, rather than the loss of within-. While it 1050 is no longer entirely clear that phoneme categories are even acquired during infancy (Feldman 1051 et al., 2021; McMurray, in press; Schatz et al., 2021), this argument demonstrates how infant 1052 results—like adult discrimination—provide little strong evidence for perceptual warping (CP).

\section{2. Methods.}

1054 In a somewhat circular development, even as infant work has argued for CP, CP is has also 1055 served as a critical underpinning of the methods used to establish this framing. Virtually, all 1056 infant methods rely on discrimination. In habituation/preference measures like the head-turn 1057 preference procedure (Jusczyk \& Aslin, 1995) or high amplitude sucking (Eimas et al., 1971), infants engage in some action (fixating a light, sucking on a pacifier) to cue a baseline stimulus

1059 (e.g., a repeating /b/). Once they habituate or are familiarized with it, this behavior is reduced.

1060 Then, the stimulus is changed. If the change is detectable, the behavior should be reengaged. 1061 Similarly, in the conditioned head-turn procedure (Kuhl, 1979), the infant hears a repeating 1062 baseline stimulus and is conditioned to look to the side whenever a change is detected. Both 1063 are measures of discrimination. In fact, there are few good ways to instantiate a labeling or identification task with babies 1065 (though see Albareda-Castellot et al., 2011), consequently discrimination is all we have. Under 1066 the assumption of $\mathrm{CP}$, this is not problematic. If discrimination is predicted by identification, we 1067 can use differences in discrimination to make inferences about categorization. A failure to 1068 discriminate two tokens that are presumably from the same category indicates categorization; 1069 successful discrimination implies two categories. However, if we cannot assume CP, what are 
1070

1071

1072

1073

1074

1075

1076

1077

1078

1079

1080

1081

1082

1083

1084

1085

1086

1087

1088

1089

1090

1091

1092

1093

1094

1095

we to make of such data?

Moreover, it is not clear that CP holds in infancy: two studies show evidence of withincategory discrimination in infancy (McMurray \& Aslin, 2005; Miller \& Eimas, 1996) and Galle and McMurray (2014) conducted a systematic review across multiple studies showing evidence for gradient sensitivity when data was pooled across studies that individually reported CP. Clearly, the differences in discrimination that have been reported are not irrelevant to categorization, but we may not be able to make strong claims that infants have speech categories. In short, we need a clearer understanding of the link between these infant discrimination measures and categorization (the derivation chain) in order to know what we know (see McMurray, in press, for further discussion of the limits of infant methods).

\section{Perceptual Narrowing.}

The dominant story of early development is a form of perceptual narrowing (Maurer \& Werker, 2014) - infants lose discriminative sensitivity to things they don't need. This is consistent with the broader meta-theoretical view of CP that the goal of perception is to filter out unnecessary variation. But given the research presented here, that view is no longer tenable. Instead, it appears that development may be more concerned with more accurately characterizing the statistical structure of speech cues and learning the various factors that give rise to the observed data. For some sounds (e.g., non-native sounds), this may mean narrowing; but for other sounds, the representation may need to be enriched (Eilers \& Minifie, 1975; Eilers et al., 1977; Narayan et al., 2010), and sensitivity may need to be improved to accurately account for contextual factors like talker. In fact, taking a lifespan approach (Figure 9D; McMurray et al., 2018), and using tools more sensitive to the structure of speech, we see that children actually improve in their ability to encode fine-grained, gradient detail over the first 18 years of life - the exact opposite of narrowing. In short, if speech perception is about harnessing variability to enable flexible behavior, we may need a new metaphor that moves us beyond narrowing.

4. Toward a new direction. Together, these arguments suggest the need to think 
1096 differently about what it is that infants know, what they are trying to achieve developmentally 1097 and how we get there. This may require moving beyond categories - which we can't even 1098 measure in infancy. For example, Feldman et al. (2021) argues that early infancy may be a 1099 time in which infants largely organize the perceptual space (e.g., the middle layer of Figure 3), 1100 and that true categorization may not come until children are older and have access to more 1101 words, articulation, and richer information (see also McMurray, in press).

1103 B. Development and Disorders in Older Children

1104 CP—both as a theory and a set of methods—has been instrumental in understanding 1105 development and communication disorders in older children. In typical development, a small but 1106 growing number of studies has used speech continua along with 2AFC tasks to demonstrate 1107 changes in phonetic categorization over the school-age years and later (e.g., Figure 9C) 1108 (Bernstein, 1983; Hazan \& Barrett, 2000; McMurray et al., 2018; Nittrouer, 1992, 2002;

1109 Slawinski \& Fitzgerald, 1998). These studies have typically not used a discrimination task to 1110 establish CP, but they nonetheless show changes in categorization. Moreover, a voluminous 1111 body of work has used similar tasks (often with a discrimination task to establish CP) in children 1112 with disorders such as dyslexia, developmental language disorder, or brain damage (e.g., Dial 1113 et al., 2019; Robertson et al., 2009; Serniclaes et al., 2004; Werker \& Tees, 1987). With respect to identification, such studies typically find that younger or impaired

1115 listeners show differences in the identification curve relative to typical or older listeners (Figure 1116 10B). These take the form of either a shallower slope (gray curve) or asymptotes that do not 1117 reach 0 or 1 . The question is what does this mean?

1118 Consider the labeling function. The typical interpretation (assuming CP) is that listeners 1119 are striving for a discrete categorization. Consequently, a shallower slope must come from 1120 noise, specifically, in encoding the continuous cue. For example, if a VOT of $15 \mathrm{msec}(\mathrm{a} / \mathrm{b} /)$ 1121 was misheard as $20 \mathrm{msec}(\mathrm{a} / \mathrm{p} /)$ on some trials, this would cross the boundary, and result in a 
1122 different identification. However, if a VOT of 0 msec was misheard as $5 \mathrm{msec}$, it would still be a

$1123 / \mathrm{b} /$ and yield no difference. In contrast, if cue encoding was not noisy, but there was noise at the

1124 level of categories, this should be fairly independent of the VOTs - all /b/'s are occasionally

1125 misperceived as /p/'s and vice versa. Consequently, if we assume CP, the interpretation is

1126 clear: a shallow slope is an unambiguous marker of noisy cue encoding, and a reduced

1127 amplitude is an unambiguous marker of a category-level difference.

1128 But what if the categories are gradient? As described, gradient categories could be

1129 beneficial—not costly—in some circumstances. However, the mapping between a gradient

1130 underlying category and 2AFC performance is ambiguous. A shallower slope could indicate a

1131 more gradient category, which might be interpreted as a rational response to uncertainty

1132 (Clayards et al., 2008). However, it could also reflect a noisier system (much like the model

1133 assumed by CP). It's possible that both are occurring: shallower slopes in some children

1134 indicate greater noise, but in other listeners may indicate a useful adaptation! The 2AFC task

1135 cannot distinguish these possibilities (Apfelbaum et al., submitted). A critical issue is how

1136 listeners choose to map an underlying gradient representation to a response across trials: if a

1137 given token is heard as $60 \% / \mathrm{b} /$-like, do they attempt to match this probability (choosing $/ \mathrm{b} / 60 \%$

1138 of the time), or do they always choose /b/ (winner take all) (Nearey \& Hogan, 1986)?

1139 Understanding this linking function could radically change our interpretation.

1140 Other studies attempt to pin a deficit specifically at the level of cue-encoding using

1141 discrimination tasks; for example, Serniclaes et al. (2004) has argued dyslexia can be linked to

1142 an inability to sufficiently ignore within-category detail, and Robertson et al. (2009) has argued

1143 that children with developmental language disorder show impaired between-category

1144 discrimination. However, given the arguments against CP at both theoretical and

1145 methodological levels, it is not clear what these claims mean. If the Pisoni and Tash (1974)

1146 model (Figure 5) is right and both category and auditory levels contribute to categorization, then

1147 perhaps these differences in discrimination simply reflect poorer phonological categorization. 
Alternatively, the kinds of discrimination tasks used in such studies heavily tap working memory, cognitive control and phonological processing. All three skills likely co-develop with speech perception or are comorbid deficits in many communication or neurological impairments.

Perhaps, then, these differences in discrimination don't reflect perception or categorization at all, but rather reflect differences in processes that are entirely external to speech categorization. Minimally, it is clear that studies of communication disorders and development should better account for what is known about the memory and decision demands of discrimination tasks (Gerrits \& Schouten, 2004). However, given the various cognitive comorbidities, as well as the uncertainty surrounding forced-choice categorization, it may be better to explore radically different methodological alternatives such as the continuous VAS tasks, eye-tracking in the VWP, or ERP components like the N1.

Beyond methodology, the implicit claim of CP is that steep categorization, and the suppression of within-category detail is the "goal" of normal development. That's what typical listeners should do; that's what children should learn to do; and that's what impaired listeners do not do as effectively. In light of more modern thinking in speech perception, that is clearly not the case. Gradiency is important-both for speech perception as classically defined and other functions (articulatory control, sociolinguistic marking). It is functionally beneficial for listeners and may be a crucial avenue by which people facing communicative impairments adapt (McMurray et al., 2016). It may be something that development or learning is attempting to achieve. In short, we need a new paradigm - both methodologically and theoretically - for thinking about these crucial real-world problems.

\section{Bilingualism and L2 Acquisition}

Work on bilingualism and second language (L2) acquisition has been motivated by CP in multiple ways. This is a voluminous body of work, and I won't attempt a systematic review. Instead, I offer two observations. 
1174 1. Critical or Sensitive Periods.

1175 First, there is a strong presumption of critical or sensitive periods for L2 acquisition. This has

1176 been motivated in part by work examining proficiency as a function of when a learner begins

1177 learning a second language (Johnson \& Newport, 1989). However, this work has also been

1178 challenged by much larger-scale studies that account for not only when L2 acquisition begins,

1179 but also for the fact that language learning (both L1 and L2) is likely to be protracted

1180 (Hartshorne et al., 2018). This seems to show a much larger sensitive window (through age 18).

1181 The other argument for a sensitive period is the apparent rapid emergence of $L 1$ speech

1182 categories in infancy (Werker \& Tees, 1984), coupled with the struggles many adult L2 learners

1183 face in acquiring such categories (Strange \& Shafer, 2008): if infancy is a special time for

1184 speech category learning, this can explain these difficulties (Werker \& Hensch, 2015).

There are two problems with this presumption. First, as I described, without the assumptions of $\mathrm{CP}$, it is challenging to conclude that infants truly have acquired speech other things (Feldman et al., 2021; McMurray, in press; Schatz et al., 2021), and development may be quite a bit slower (Hazan \& Barrett, 2000; McMurray et al., 2018) (supporting the Hartshorne et al., 2018, analysis). Second, infant methods are radically different than from those used with adults; a typical infant study may present infants with multiple tokens of the two

1192 sounds to be discriminated, and any difference in listening time is used to support

1193 discrimination. In contrast, adults get one token/trial, and the criterion is accuracy. If adult L2

1194 learners were tested with the relatively looser infant procedures, would they look just as good?

1195 This challenges the basic assumption that L2 learning adults struggle, while L1 learning infants

1196 "get it." Both of these arguments undercut the premise of a critical period and call for more

1197 rigorous and direct empirical evaluation of this construct (Fuhrmeister et al., 2020) as well as

1198 new ways of thinking about critical periods (Thiessen et al., 2016).

\section{$1199 \quad 2 . \quad$ What is the goal of bilingual speech perception?}


1200 Most work on bilingual or L2 speech perception implicitly assumes CP as a framing. This could 1201 restrict interpretation along two dimensions. First, as with children and impaired populations, it is 1202 not warranted to presume that a sharp categorical boundary is desirable. Gradiency could be 1203 helpful in general, and, in a multi-lingual environment, gradiency may be even more helpful.

1204 When confronted with multiple languages or dialects (potentially changing from moment to

1205 moment), gradiency may be needed to be more flexible and to incorporate contextual

1206 information (about the language or dialect spoken). Thus, the question should not be how 1207 listeners attain sharp boundaries, but how they attain flexibility.

1208 Second, CP highly emphasizes boundaries. In a bilingual situation, that frames 1209 questions such as whether a Spanish/English bilingual puts their VOT boundary at $20 \mathrm{msec}$ 1210 (English) or $0 \mathrm{msec}$ (Spanish) and whether they can shift it rapidly. But no serious model of 1211 speech perception assumes boundaries anymore. Instead, prototypes (e.g., Figure 8) (or some 1212 functional variant of that) are the norm. But crucially, prototypes need not be mutually exclusive. 1213 A listener could have an English /b/ category centered at $5 \mathrm{msec}$, and a Spanish /b/ category 1214 centered at $-60 \mathrm{msec}$. Thus, moving beyond CP may pose new questions for work on 1215 bilingualism and L2 acquisition.

\section{Other domains of cognition and perception}

1218 As I described, the extension of CP to domains beyond speech perception has been an active 1219 endeavor, and it has played an important role in debates about linguistic relativity (Franklin et 1220 al., 2008), modularity (McKone et al., 2001), and other fundamental issues. While it is beyond 1221 the scope of this review to fully address this problem, it is worth a few brief comments.

1222 First, for the most part, this literature outside of speech has assumed CP and made little 1223 contact with the literature specifically on CP within speech. There are exceptions, but often the 1224 deeper message is lost. Goldstone and Hendrickson's influential (2010) review does discuss 1225 some of this literature, but it does not appear to lead them to question the premise. Even the 
1226 original demonstration of categorical perception in color (Bornstein \& Korda, 1984) explicitly

1227 adopts the Pisoni and Tash (1974) model (Figure 3), but fails to realize its deeper implications.

1228 Second, the few studies that have addressed this issue head on suggest a story much

1229 more consistent with non-categorical perception. For example, Hanley and Roberson (2011)

1230 conducted a retrospective analysis of several studies of face and color CP and found that the

1231 order in which the stimuli were presented in the discrimination task had a major impact on

1232 performance, and when that was accounted for, results were well described by a model

1233 featuring the continuous encoding of the input, but in which categories could also impact

1234 discrimination (e.g., Pisoni \& Tash, 1974). Similarly, Roberson et al. (2009) conducted a serious

1235 analysis of the discrimination tasks used to establish color perception (analagous to Gerrits \&

1236 Schouten, 2004; Pollack \& Pisoni, 1971), and identified a less biased task in which colors were

1237 presented immediately adjacent to each other. When this task was adopted, the CP effect

1238 disappeared. While these sorts of issues are still under active debate (e.g., Best \& Goldstone,

1239 2019), this suggests some movement in the broader field.

1240

Third, a key argument here is that the claims of CP do not live in isolation from broader

1241 theories of speech perception-CP makes claims that the rest of the system must live with. In

1242 speech, throwing away fine-grained continuous detail may pose limits on the degree to which

1243 the system has access to this detail for other things like integrating cues over time, using the

1244 same cue for multiple purposes or identifying sociolinguistic properties. This kind of theoretical

1245 inconsistency may arise in other domains of cognition where CP is invoked.

1246 Face perception offers a useful illustration. In this domain, there has been rapid adoption

1247 of what's termed "norm-based" face coding (Leopold et al., 2001; Rhodes \& Leopold, 2011). In

1248 this view, face perception is seen as dimensional, and frequent experience with classes of faces

1249 can shift the mean of this dimension - the same operation posited by C-CuRE in speech for

1250 talker compensation (McMurray \& Jongman, 2011). Critically, this kind of dimensional shifting

1251 cannot occur if dimensions are collapsed into discontinuous regions. In a related issue, virtually 
1252 all domains of perception confront the problem identified by Mermelstein (1978): that the same 1253 continuous dimension may be needed for more than one distinction. It may make sense to 1254 minimize within-category differences in color if the only goal is accurate labeling. But people 1255 must also use subtle aspects of color to judge attractiveness, to compensate for ambient 1256 lighting, or many other things. In fact, color labeling may be one of the least important goals of 1257 color perception! Similarly, if variation within various exemplars of the same face is ignored, how 1258 can an observer use subtle differences in that face to judge facial emotion or health? One can 1259 argue that perhaps CP-like effects emerge in the context of a particular task, but this then is a 1260 far cry from everyday cognition and the radical warping posited by CP.

\section{E. Summary}

1263 As the foregoing discussion reveals, in development, disorders, multilingualism and cognition 1264 more broadly, a strong presumption of CP has shaped many things, from the tasks used to 1265 assess perception and categorization, to the interpretation of the functional goals of the system. With the demise of CP within speech perception this has not been questioned. It needs to be. of cognition to an experiment or measure. In all of these domains, CP was an essential piece of

1270 the derivation chain. However, CP is now a part of the past; it makes problematic theoretical 1271 assumptions, and we better understand discrimination tasks. This undercuts the derivation 1272 chains used in these lines of work and calls for a new approach.

\section{Where do we go from here?}

1276 CP has been with us since before the cognitive revolution, and before I was born. Despite 1277 ample empirical evidence to the contrary, it remains. Despite the fact that it has no answer to 
1278 fundamental facts about speech (like the fact that speech cues do multiple things), it remains.

1279 Despite the fact that its assumptions are inconsistent with most modern theories of speech

1280 perception, it remains. Why? What are we to learn? Where do we go from here? It helps to start

1281 by considering why it was (and remains) compelling.

\section{A. What made (and makes) CP so compelling?}

When CP was discovered and promulgated, speech science was in a different place. While the

1285 early work identified many key sources of variability (Delattre et al., 1955), we had not identified

1286 the hundreds of phonetic cues we know of now, we did not have access to large corpora, and

1287 statistical tools for pooling "big data" were not in wide use. Speech perception for many was

1288 more of an analytic_-rather than synthetic_-process focusing on a small number of cues in 1289 isolated (simplified) syllables and so forth (thought see Blumstein \& Stevens, 1979). And the problem was narrowly defined in terms of categorization (much as l've simplified here). In this light, it is easy to see how CP would be an attractive solution (see also Supplement S2). problem for the first time now, with access to large corpora and tools for integrating dozens of measurements, with far more phonetic cues, with much more variable speakers and listeners under investigation, and with a richer understanding of related processes like word recognition 1296 and indexical perception? Would we ever have come up with CP? It's not clear that we would 1297 have. Historical precedence is not an argument for retention. meta-expectations about language. Any ordinary person can tell you, there is nothing in 1300 between a bunt and a punt-these are distinct words. And for speakers of an alphabetic 1301 language, there may be nothing between a /b/ and a /p/. Arguably our over-reliance on the IPA 1302 as a tool for conceptualizing sound contributes as well-well before we had CP, field work in 1303 linguisics, speech pathology and developmental work was focusing on a symbolic 
1304 representation of a fundamentally continuous signal CP matches these intuitions of

1305 discreteness. That is, we want to be able to talk about sound in terms of categories, and we

1306 even think we hear it in terms of categories. But this is an illusion-when we look closely with

1307 unbiased discrimination tasks or EEG we see a different story.

1308 People like to categorize—whether in terms of statistical significance, gender categories

1309 or color-and are uncomfortable with gradience and nuance as they are difficult to verbalize.

1310 CP would seem to take something that is quintessentially messy and nuanced-perception-

1311 and offers a comforting answer that it is not, that categories are just fine.

\section{B. What gives? Why is CP still with us?}

1314 The scientific community is generally more comfortable with nuance. And within the community

1315 of researchers working directly on CP, we have clearly known for a long time that speech is not

1316 perceived categorically. So how has this persisted? This is illustrated anecdotally by an

1317 experience I had as an early graduate student. When I submitted the first few papers arguing for 1318 gradiency for publication (McMurray, Aslin, et al., 2008; McMurray et al., 2002), the reviewers

1319 argued that my work was setting up a straw man, that CP was over, and we should move on.

1320 Clearly, the reviewers knew what was up. And yet this message did not appear to be true.

1321 Almost everyone outside of speech perception (e.g., in vision) was still assuming CP, and even

1322 within multiple areas of speech perception, it was still with us

1323 That's still true today, 20 years later. Work is building new theories of CP even in the

1324 field of speech perception (Kronrod et al., 2016), and in broader areas of cognition (Feldman,

1325 2021; Zhang et al., 2021). CP continues to be applied in a variety of subdomains of speech

1326 perception such as L2 learning and language/hearing disorders ${ }^{4}$. CP is not going away, despite

\footnotetext{
${ }^{4}$ As evidence: A google/scholar search of ("categorical perception" AND "L2 learning") led to 1170 hits since 2017, and ("categorical perception" AND "communication disorders") led to 3020! Many of these papers may simply be using the phrase categorical perception to refer to categorization (not sensory discontinuities), but these impressive results suggest this idea is still omnipresent.
} 
1327 the fact that the small community of hardcore speech nerds who have worked directly on CP

1328 knows better. Bearing witness to this is the continual appearance of major reviews and large 1329 scale studies of CP (Goldstone \& Hendrickson, 2010; Kronrod et al., 2016) that treat CP as a

1330 fundamental fact to be explained. While some of them do make contact with the literature 1331 reviewed here (e.g., Goldstone \& Hendrickson, 2010), they do not question the premise.

1332 I don't know how those of us who have worked on CP could make the truth more 1333 apparent. There are papers with titles like The End of Categorical Perception as We Know it 1334 (Schouten et al., 2003), Categorical results do not imply Categorical Perception (Hary \& 1335 Massaro, 1982), and my favorite, Categorical Perception of speech: A largely dead horse, 1336 surpassingly well kicked (Crowder, 1989). Could speech perception researchers be any more direct? One does not even need to read the papers to know that something is up ${ }^{5}$.

1339 of the meta-narrative of speech, a scientific meme. It is easy to dismiss individual papers here 1340 and there, while at the same time treating them as exceptions to a general rule. I hope what this 1341 review has done is to show how there are simply too many inconsistencies to hold on to CP 1342 anymore. The question is where to go from here (see Box 2 for concrete steps).

\section{Science Communication}

1345 Part of the solution has to be better communication. There are few, if any, reviews of CP in the 1346 speech literature (broadly construed) that wrestle with the more modern conception. The most 1347 comprehensive recent reviews that l've seen come from outside of speech (Goldstone \&

1348 Hendrickson, 2010). We—speech researchers of all sub-domains—must take it upon ourselves 1349 to communicate outside our field. We can't expect textbook authors and people outside of our 1350 field to figure it out from more highly technical papers in the Journal of the Acoustical Society of

\footnotetext{
${ }^{5}$ And here l've put the titles right in the text to save you the trouble of checking the references. Although since JASA puts notes at the end of the manuscript, you just did that too.
} 
1351 America (yes, I get the irony). If my review was overly pointed, it was for this purpose-subtlety 1352 will not help make a dent in the rapid growth of CP (Figure 1).

1353 In fact, even the basic findings are not making it out of our field. Many people in use the 1354 term categorical perception to refer to any task in which people identify tokens from a 1355 continuum, whether or not discrimination is measured, and whether or not the researcher is 1356 making claims about the perception of the continuous space. People commonly refer to a 1357 sigmoidal identification function as a "categorical perception" curve - even without 1358 discrimination or any claim about perception. How do so many papers that mischaracterize or 1359 misapply CP continue to be published? Have speech researchers in the categorization 1360 community agreed to just let this pass? Are they not reviewing these manuscripts? A critical aspect of this issue is teaching. I recently surveyed most of the major 1362 psycholinguistics textbooks, and many phonetics texts. All treat CP uncritically. I suspect it's the 1363 same in other fields such as speech and hearing science or phonetics (when speech 1364 categorization is covered at all). Why is that? The easy response is that the alternative is too complicated for undergraduates. But this sells our students short. The debates around CP are an excellent opportunity to teach how science can be self-correcting, and how theories of 1367 speech perception need to account for fundamental properties of the speech signal. My 1368 suspicion is that many of us are making end runs around the texts to teach CP correctly with 1369 innovative demos and tools. If so, these should be shared. Moreover, too many textbooks treat 1370 speech perception as an interesting set of phenomena for students to share with their parents:

1371 Categorical Perception, the McGurk effect, Duplex Perception, the Cocktail Party Effect. That's 1372 important for raising interest, but it doesn't get our students closer to a deeper understanding of 1373 how speech and language works. We should be teaching students how the auditory system 1374 actually processes speech. The gradient alternative is not too complex: use multiple cues, 1375 engage in simple forms of compensation, maintain a prototype structure to be flexible, and 1376 constantly learn and update. This should be a message we can tell clearly. 


\section{Throwing out prior results with the bathwater?}

1379 A question now is what to make of the voluminous findings that actually report the characteristic 1380 profile of between- and within-category discrimination that constitutes CP. It is tempting to 1381 dismiss them out of hand; but that's wrong. The data is real, even if the premise is problematic. 1382 So, we need some framework for understanding these prior results, and for making sense of 1383 differences in CP-like behavior across conditions and groups.

Clearly, the wrong conclusion is that perception is warped by the presence of categories.

1385 That is a not tenable claim anymore. However, a not unreasonable claim - following Figure 5 may be that once CP (empirically) is observed, categorization must be pretty robust (fast and strong) in order to contribute to the discrimination judgement-weakly represented or acquired categories are simply not engaged in discrimination. This accords well with the learning accounts-when CP is not observed in L2 learning (Miyawaki et al., 1975), listeners simply have categories that are represented or accessed less robustly. If we restrict ourselves to the idea that $\mathrm{CP}$ reflects robust categorization, this seems reasonable. However, perhaps we should just

1392 call it categorization (or perception of categories), not categorical perception (Holt \& Lotto, 2010). And perhaps future work should skip the discrimination tasks more generally—there are more informative ways to assess categorization.

The alternative approach is to restrict the domain of interpretation. CP is not a paradigm

1396 for understanding perception of the auditory space, but it is a useful way to think about how 1397 people engage in discrimination judgements. Discrimination is an interesting human behavior 1398 and arguably important in its own right. Maybe CP is simply a way of probing the influence of 1399 higher-level knowledge on discrimination, and of probing various memory biases. If that's the 1400 question, perhaps $\mathrm{AX}$ and $\mathrm{ABX}$ task serve an important role. Again, this is defensible, but again 1401 it's not quite CP. Perhaps this is biased discrimination? In both approaches, the key is to have a clear understanding of what we're actually 
studying in CP paradigms so we know what can be concluded. What does not seem reasonable to me is to stretch this concept in every conceivable direction to try to preserve the name.

\section{E. Methods?}

The debates over CP and the development of this line of work clearly point to the need for a stronger understanding of methods. The limits of discrimination tasks are clearly highlighted (Pisoni \& Lazarus, 1974; Schouten et al., 2003) and researchers need to be aware of these limits. The basic properties and concerns about discrimination tasks should be well known in any field that relies on them.

But the 2AFC task is not without its issues either. As our brief discussion about development reveals, there are limits of interpretation to this task: is a shallow slope a natural reflection of a gradient system, or the result of a noisy but categorical system? These things cannot be known from this task alone. In fact, even if listeners have an underlyingly gradient boundary, they could still show a steep slope if their response on each trial always reflects the more likely of the two alternatives (Nearey \& Hogan, 1986). We simply do not understand the derivation chain (Meehl, 1990) linking an underlying category mapping to people's behavior in this highly simple task. But even if we did, this task may simply be underdetermined. More sophisticated measures are needed. But these need not be technically sophisticated (e.g., eye-tracking or ERPs). As Massaro, Miller and Kapnoula have shown (Kapnoula, 2015; Massaro \& Cohen, 1983; Miller, 1997), even something as simple as a continuous rating task (Figure 6), can dramatically open up new possibilities for understanding categorization. More sophisticated tools like eye-tracking and ERPs are not going to be a panacea. The most common ERP measure of speech, for example, the mismatch negativity (see Phillips, 2001, for an example applied to CP), is in fact a measure of discrimination, with all the caveats to interpretation that apply to any discrimination task. Techniques like ERP and the VWP are useful, but they need to be deployed in ways that are linked to the underlying cognitive 
1429

1430

1431

1432

1433

1434

1435

1436

1437

1438

1439

1440

1441

1442

1443

1444

1445

1446

1447

1448

1449

1450

1451

1452

1453

1454

and perceptual operations, and that take advantage of their own unique properties. That is, they need to be deployed in ways that respect the derivation chain. For example, the ability to condition analysis of ERPs or eye-movements on the overt response offers the unique ability to ensure that one is examining within-category variation; or the fact that the $\mathrm{N} 1$ is tied to early auditory cortical regions offers an ability to isolate processing of continuous acoustic encoding.

Third, CP also introduced to us the notion of a speech continuum. This is arguably the most valuable and defensible methodological innovation. Nothing in the foregoing review invalidates this approach, and the fact that it can span an ambiguous region can highlight subtle but theoretically meaningful effects that cannot be seen elsewhere. However, we can improve it. It would be helpful to standardize how continua are reported and used (see Supplement 1). This can help ground arbitrary step numbers to real acoustic measurements. Such standardization can help compare effects across different contrasts and could be crucial for the currently fashionable meta-analytic approaches. But as the tools for constructing continua become better and easier, we must also be careful that continua are phonetically realistic —-they span neighboring phonemes (not passing through a third phoneme or a dead space) and manipulate cues in ways that reflect the variation that real articulators are capable of. And this is not a panacea, nor is it the only way to study speech (note to self).

We should also consider moving beyond continua. In the era of big data and large-scale statistical models, one can (for example) simply record dozens of naturalistic exemplars, measure their phonetic properties, and give them (unmanipulated) to listeners to categorize. Then, logistic models can sort out which phonetic properties are contributing to a given percept or how that changes across experimental conditions (McMurray \& Jongman, 2011). This could provide a powerful—and phonetically grounded—complement to continua.

The last methodological concern is the biggest. Many experiments are predicated on some notion of what kind of processing is typical, efficient, optimal, or ideal. This is perhaps the most serious impact of $\mathrm{CP}$, by biasing researchers to expect sharp categorization and poor 
1455 within-category discrimination as the ideal. Instead, modern theories of speech suggest a 1456 system that is much more gradient, flexible, and complex, and our thinking needs to adapt.

\section{F. Conclusions}

1459 CP have long lived. In some ways, it is easy to work around many of the points raised here 1460 individually. I've argued that CP is incompatible with the kind of rich compensation needed to 1461 account for talker and coarticulatory variation. An easy counterargument that could preserve CP 1462 would be that listeners do all this before they do CP (or maybe these processes are how one 1463 becomes categorical). But if that's the case, what's the point of adding CP to the system (and in 1464 that case, is this really perception)? In fact, attempting to salvage CP in this way ignores the 1465 fundamental claim of CP that the system is attempting to suppress variation - instead, it seems 1466 to be harnessing it. One could also attempt to account for the myriad discrimination results by 1467 claiming that there's an early categorical representation alongside a linear one. But is that really 1468 any different than the Pisoni and Tash (1974) model?

1469 The point is, if one contorts oneself, we can salvage CP from many of these attacks. But 1470 at some point, we have to look at the bigger picture: CP as an empirical finding or a theory is 1471 just not logically consistent as a whole. It is not a coherent account of the extant data nor of 1472 speech perception, and its theoretical claims are incommensurate with theories of speech that 1473 use the fine-grained detail that CP would discard to do useful work for listeners. Given the 1474 wealth of empirical evidence against CP and the strong theories of speech that either do not 1475 require it or conflict with it, CP no longer adds to the greater coherence of our understanding of 1476 speech perception. Can we be done?

\section{Acknowledgements}

1479 The author would like to thank Keith Apfelbaum, Joe Toscano, Joan Sereno and Allard 1480 Jongman for helpful comments on this manuscript; Ethan Kutlu for engaging ideas about multi1481 lingualism, and Effie Kapnoula for ideas regarding individual differences. He would particularly 
1482 like to thank Ben Munson whose thoughtful review of this manuscript led to a rethinking of 1483 crucial issues around the relationships among fields, and the importance of social and other 1484 functions of speech. This project was supported by DC008089. 


\section{Inset Boxes}

\section{Box 1: What counts as steep? And what is a step?}

1488

1489

1490

1491

1492

1493

1494

1495

1496

1497

1498

1499

1500

1501

1502

1503

1504

1505

1506

1507

1508

1509

1510

1511

1512

1513

1514

1515

1516

1517

1518

1519

1520

1521

1522

1523

1524

1525

1526

1527

1528

1529

1530

$\mathrm{CP}$ is often colloquially defined as a "sharp" categorization function across a continuum that varies in some number of small steps. However, this can be illusory and subjective. If a researcher restricts the range of a continuum to a narrow region around the boundary (and then uses a small step size), the function might look quite gradient; while an artificially expanded range could make a relatively gradient function look step-like (c.f., McClelland \& Elman, 1986, p. 46). The field has not converged on standards for continuum generation that would allow any kind of objective statement.

Here, I propose a simple solution. It is tempting to try to equate steps at a psychophysical level - much like the Munsell scale has done for color. That is, could we use psychophysical discrimination thresholds to decide how many msec of VOT equals $10 \mathrm{~Hz}$ of F1? However, speech contrasts are often highly multi-dimensional (Jongman et al., 2000; Lisker, 1986; Repp, 1984), and individual cues may be nonlinearly represented. Instead, I suggest a top-down approach, starting from the phonetic data.

1) Start with the actual words or strings that will be "morphed" into the continuum.

2) Generate recordings of multiple exemplars of each of them. These could be from the same talker or from a representative group of talkers.

3) Identify the relevant cues from the phonetic literature and measure them in the recordings.

4) Use the mean of those cue values as the prototype "locations" in a high-dimensional space.

5) Generate the continuum based on some subset of the recordings.

6) Finally, measure the cue values for the generated stimuli - these can be projected onto the line connecting the two prototypes to convert step number to a proportion of distance between the phonetic prototypes. Values more extreme than the endpoints (e.g., a super long VOT) could be treated as greater than $100 \%$ or less than $0 \%$.

It may be helpful for labs to begin standardizing how they report continua in this way. This would permit meta-analyses, as well as more objective measures of steepness (e.g., change in proportion responses / step is now constant across all studies), which could eventually be used to establish criteria. See Online Supplement for equations and a worked example.

\section{Box 2: Speech perception in a Post-CP world}

$\mathrm{CP}$ has shaped the field for so long that it is unclear what the field will look like without it. To start that conversation, here are some concrete (but not exhaustive) recommendations.

\section{Science Communication}

- Textbooks should be revised to reflect the modern view.

- Pithy review articles are needed to communicate to speech-adjacent fields.

- Reviewers need to be more pointed in challenging appropriate uses of CP.

\section{Methods}

- Small differences in discrimination tasks can lead to different memory demands and different levels of bias. We cannot conclude CP from discrimination tasks, and they should be used very carefully. 
1531

1532

1533

1534

1535

1536

1537

1538

1539

1540

1541

1542

1543

1544

1545

1546

1547

1548

1549

1550

1551

1552

1553

1554

1555

1556
- Forced-choice identification tasks may be problematic when studying the slope of the identification function (e.g., over development) (Apfelbaum et al., submitted). For this issue, a continuous VAS task may be better.

- Forced-choice identification tasks are not problematic for assessing boundary shifts (e.g., trading relations, perceptual learning paradigms).

- Measures that allow researchers to simultaneously measure the category label and the underlying activation on the same trial (e.g., specific VWP or ERP paradigms McMurray et al., 2002) are valuable.

- Speech continua are valuable when they are constructed on the basis of phonetic insight (not arbitrary morphing). It would help to standardize them across dimensions.

- Speech continua should be complemented with more naturalistic methods in which actual recordings are measured along multiple dimensions to relate categorization to actual acoustic variance.

\section{Theory and Interpretation}

- The focus should be on theories of perception not theories of categorization along a speech continuum.

- We need stronger theories of the problems highlighted by modern ideas: how do listeners achieve flexibility? Where do individual differences come from? How do listeners use finegrained acoustic variability?

- Theoretical accounts of development, bilingualism, and clinical populations should abandon the premise that the goal of speech perception is discrete categorization and ignoring withincategory detail, and instead ask how these listeners achieve a gradient, flexible and efficient categorization. 
Figure 1: Number of new citations in consecutive 5-year periods starting in 1955. Search was conducted on Google/Scholar with the term "categorical perception"

Figure 2: Canonical results of a categorical perception experiment. On the $\mathrm{X}$ axis, step refers to step from a speech continuum, where step 1 might be a prototypical /b/ and step 10, a prototypical $/ \mathrm{p} /$. Identification (dashed line, left axis) is coded as the proportion of responses that match one of the endpoints. Here, 0 is $/ \mathrm{b} /$ and 1.0 is $/ \mathrm{p} /$, so that $0 \%$ responding indicates the participant consistently heard /b/. Discrimination is assessed between neighboring points (e.g., between step 1 and 2, 2, and 3, and so forth). The peak indicates that discrimination is better between steps 5 and 6 (spanning the boundary shown by the identification results) than

1568 between steps 1 and 2 (both /b/).

Figure 3: A rough conceptual model of the speech chain assumed by CP. Speech input is first mapped to a continuous auditory encoding that represents something like continuous cuevalues. This space is then carved into categories.

Figure 4: A) Spectral mean and F2 onset frequencies for /s/ and /J/ from Jongman et al. (2000).

The line represents a linear discriminant analysis that can separate the categories at about $92 \%$. B) A simulation of CP generated by assuming the linear boundary in A, and then doubling the distance between tokens on each side of the boundary and halving the variation within each side of the boundary; C) A simulation of CP generated by only increasing the distance, without adjusting within-category variation. D) Same data after compensating for talker and vowel differences using the regression approach of C-CuRE (McMurray \& Jongman, 2011); the discriminant analysis achieves $96.6 \%$ accuracy.

Figure 5: The Pisoni and Tash (1974) model of discrimination. Speech is transduced to a continuous perceptual space, then to categories and words (left branch). Discrimination, however, can use either the perceptual or category representation (right branch). Under these assumptions, between-category contrasts should almost always be more discriminable than within (embedded table), even if the perceptual encoding is continuous.

Figure 6: Distribution of rating scale responses for individual subjects as a function of continuum step (each row) from Massaro and Cohen (1983) (estimated from their Figure 7). A) Under a categorical model, listeners should use only the ends of the scale, and should only vary in how frequently they choose the $/ \mathrm{b} /$ or $/ \mathrm{p} /$ ends. B) Under a continuous model, the mean rating should shift with step. C) Estimated data from a typical listener.

Figure 7: Results from (Toscano et al., 2010). A) Voltage at front-central channels as a function of time and VOT. The large negative deflection at $100 \mathrm{msec}$ is the N1 and its depth is related to VOT. B) Mean N1 amplitude as a function of VOT. 
1599 Figure 8: Typical results of phoneme goodness rating experiments of Miller and colleagues

1600 (data estimated from Miller \& Volaitis, 1989). A) /p/ goodness as a function of VOT and

1601 speaking rate (the length of the following vowel). B) Phonetic measurements showing the

1602 distribution of VOT as a function of speaking rate.

1603

1604 Figure 9: Results of VWP experiments documenting gradient within-category sensitivity to fine1605 grained detail. A) Fixations to the competitor as a function of time, and distance from the category for tokens on the voiced side of the continuum from McMurray et al. (2002). B) Average fixation from 200 to $2200 \mathrm{msec}$ as a function of relative VOT in that study. C) 2AFC labeling function for children in McMurray et al. (2018); here, identification becomes steeper (more categorical) with development. D) In contrast, competitor fixations as a function of relative VOT show increasing sensitivity with development.

1610

1611

1612

Figure 10: A) Schematic results of a typical trading relations experiment examining voicing

1613 categorization (/b/ vs. /p/) as a function of VOT (x-axis) and F0 (separate curves). B) Schematic

1614 results of an experiment examining voicing categorization in younger and older children, or in

1615 disordered and typical children.

1616 
1618

1619

1620

1621

1622

1623

1624

1625

1626

1627

1628

1629

1630

1631

1632

1633

1634

1635

1636

1637

1638

1639

1640

1641

1642

1643

1644

1645

1646

References

Albareda-Castellot, B., Pons, F., \& Sebastián-Gallés, N. (2011). The acquisition of phonetic categories in bilingual infants: New data from an anticipatory eye movement paradigm. Developmental Science, 14(2), 395-401.

Allen, J. S., Miller, J. L., \& DeSteno, D. (2003). Individual Talker Differences in Voice-Onset-Time. Journal of the Acoustical Society of America, 113(1), 544-552.

Anderson, J. A., Silverstein, J. W., Ritz, S. A., \& Jones, R. S. (1977). Distinctive features, categorical perception, and probability learning: some applications of a neural model. Psychological Review, 84(5), 413.

Andruski, J. E., Blumstein, S. E., \& Burton, M. W. (1994). The effect of subphonetic differences on lexical access. Cognition, 52, 163-187.

Apfelbaum, K. S., Kutlu, E., McMurray, B., \& Kapnoula, E. (submitted). Don't Force It! Gradient Speech Categorization Calls for Continuous Categorization Tasks. Journal of the Acoustical Society of America, https://psyarxiv.com/7w93f/.

Beale, J. M., \& Keil, F. C. (1995). Categorical effects in the perception of faces. Cognition, 57(3), 217239.

Bernstein, L. E. (1983). Perceptual development for labeling words varying in voice onset time and fundamental-frequency. Journal of Phonetics, 11(4), 383-393.

Best, R. M., \& Goldstone, R. L. (2019). Bias to (and away from) the extreme: Comparing two models of categorical perception effects. Journal of Experimental Psychology: Learning, Memory, and Cognition, 45(7), 1166-1176. https://doi.org/10.1037/xlm0000609

Blumstein, S. E., \& Stevens, K. N. (1979). Acoustic invariance in speech production: Evidence from measurements of the spectral characteristics of stop consonants. Journal of the Acoustical Society of America, 66(4), 1001-1017.

Bornstein, M. H., \& Korda, N. O. (1984). Discrimination and matching within and between hues measured by reaction times: Some implications for categorical perception and levels of information processing. Psychological Research, 46(3), 207-222. 
1647 1648

Brady, S. A., \& Darwin, C. (1978). Range effect in the perception of voicing. The Journal of the Acoustical Society of America, 63(5), 1556-1558.

Brown-Schmidt, S., \& Toscano, J. C. (2017). Gradient acoustic information induces long-lasting referential uncertainty in short discourses. Language, Cognition and Neuroscience, 32(10), 1211-1228.

Carney, A. E., Widin, G. P., \& Viemeister, N. F. (1977). Non categorical perception of stop consonants differing in VOT. Journal of the Acoustical Society of America, 62, 961-970.

Chang, E. F., Rieger, J., Johnson, K. D., Berger, M. S., Barbaro, N. M., \& R.T., K. (2010). Categorical speech representation in the superior temporal gyrus. Nature Neuroscience, 13(11), 1428-1432.

Chomsky, N. (1959). A review of B. F. Skinner's Verbal Behavior. Language, 35(1), 26-58.

\section{https://doi.org/10.2307/411334}

Clayards, M., Tanenhaus, M. K., Aslin, R. N., \& Jacobs, R. A. (2008). Perception of speech reflects optimal use of probabilistic speech cues. Cognition, 108(3), 804-809.

Cole, J. S., Linebaugh, G., Munson, C., \& McMurray, B. (2010). Unmasking the acoustic effects of vowelto-vowel coarticulation: A statistical modeling approach. Journal of Phonetics, 38(2), 167-184.

Crowder, R. G. (1989). Categorical perception of speech: A largely dead horse, surpassingly well kicked. Behavioral and Brain Sciences, 12(04), 760-760.

Cutting, J. E., \& Rosner, B. S. (1974). Categories and boundaries in speech and music. Perception \& Psychophysics, 16(3), 564-570. https://doi.org/10.3758/BF03198588

Daniloff, R., \& Moll, K. (1968). Coarticulation of Lip Rounding. Journal of Speech, Language, and Hearing Research, 11(4), 707-721. https://doi.org/10.1044/jshr.1104.707

Delattre, P., Liberman, A. M., \& Cooper, F. S. (1955). Acoustic loci and transitional cues for consonants. Journal of the Acoustical Society of America, 27, 769-773.

Dial, H. R., McMurray, B., \& Martin, R. C. (2019). Lexical processing depends on sublexical processing: Evidence from the visual world paradigm and aphasia. Attention, Perception, \& Psychophysics, 81(4), 1047-1064.

Eilers, R. E., \& Minifie, F. (1975). Fricative discrimination in early infancy. Journal of Speech and Hearing Research, 18(1), 158-167. 
1674 Eilers, R. E., Wilson, W., \& Moore, J. (1977). Developmental changes in speech discrimination in infants.

1675

1676

1677

1678

1679

1680

1681

1682

1683

1684

1685

1686

1687

1688

1689

1690

1691

1692

1693

1694

1695

1696

1697

1698

1699

1700 Perception \& Psychophysics, 16, 513-521.

Eimas, P. D., Siqueland, E. R., Jusczyk, P., \& Vigorito, J. (1971). Speech perception in infants. Science(171), 303-306.

Escudero, P., Benders, T., \& Wanrooij, K. (2011). Enhanced bimodal distributions facilitate the learning of second language vowels. The Journal of the Acoustical Society of America, 130(4), EL206EL212. https://doi.org/10.1121/1.3629144

Escudero, P., \& Williams, D. (2014). Distributional learning has immediate and long-lasting effects. Cognition, 133(2), 408-413. https://doi.org/https://doi.org/10.1016/i.cognition.2014.07.002

Falandays, J. B., Brown-Schmidt, S., \& Toscano, J. C. (2020). Long-lasting gradient activation of referents during spoken language processing. Journal of Memory and Language, 112.

Feldman, J. (2021). Mutual Information and Categorical Perception. Psychological Science, 32(8), 12981310. https://doi.org/10.1177/0956797621996663

Feldman, N. H., Goldwater, S., Dupoux, E., \& Schatz, T. (2021). Do Infants Really Learn Phonetic Categories? Open Mind, 5, 113-131. https://doi.org/10.1162/opmi a 00046

Francis, A. L., Ciocca, V., \& Chit Ng, B. K. (2003). On the (non)categorical perception of lexical tones. Perception \& Psychophysics, 65(7), 1029-1044. https://doi.org/10.3758/BF03194832

Franklin, A., Drivonikou, G. V., Clifford, A., Kay, P., Regier, T., \& Davies, I. R. L. (2008). Lateralization of categorical perception of color changes with color term acquisition. Proceedings of the National Academy of Sciences, 105(47), 18221-18225. https://doi.org/10.1073/pnas.0809952105

Freedman, D. J., Riesenhuber, M., Poggio, T., \& Miller, E. K. (2001). Categorical Representation of Visual Stimuli in the Primate Prefrontal Cortex. Science, 291(5502), 312-316. https://doi.org/doi:10.1126/science.291.5502.312

Fry, D. B., Abramson, A. S., Eimas, P. D., \& Liberman, A. M. (1962). The identification and discrimination of synthetic vowels. Language and Speech, 5, 171-189.

Frye, R. E., McGraw Fisher, J., Cody, A., Zarella, M., Liederman, J., \& Halgren, E. (2007). Linear coding of voice onset time. Journal of Cognitive Neuroscience, 19, 1476-1487. 
The Myth of Categorical Perception

1701 Fuhrmeister, P., Schlemmer, B., \& Myers, E. B. (2020). Adults Show Initial Advantages Over Children in

1702

1703

1704

1705

1706

1707

1708

1709

1710

1711

1712

1713

1714

1715

1716

1717

1718

1719

1720

1721

1722

1723

1724

1725

1726

Learning Difficult Nonnative Speech Sounds. Journal of Speech, Language, and Hearing Research, 63(8), 2667-2679. https://doi.org/doi:10.1044/2020 JSLHR-19-00358

Gaißert, N., Waterkamp, S., Fleming, R. W., \& Bülthoff, I. (2012). Haptic Categorical Perception of Shape. PLoS ONE, 7(8), e43062. https://doi.org/10.1371/journal.pone.0043062

Galle, M. E., Klein-Packard, J., Schreiber, K., \& McMurray, B. (2019). What Are You Waiting For? RealTime Integration of Cues for Fricatives Suggests Encapsulated Auditory Memory. Cognitive Science, 43(1), e12700. https://doi.org/10.1111/cogs.12700

Galle, M. E., \& McMurray, B. (2014). The development of voicing categories: A meta-analysis of 40 years of infant research. Psychonomic Bulletin and Review, 21(4), 884-906.

Gauthier, B., Shi, R., \& Xu, Y. (2007). Learning phonetic categories by tracking movements. Cognition, 103(1), 80-106. https://doi.org/https://doi.org/10.1016/j.cognition.2006.03.002

Gerrits, E., \& Schouten, M. E. H. (2004). Categorical perception depends on the discrimination task. Perception \& Psychophysics, 66(3), 363-376.

Getz, L. M., \& Toscano, J. C. (2021). The time-course of speech perception revealed by temporallysensitive neural measures. Wiley Interdisciplinary Reviews: Cognitive Science, 12(2), e1541.

Goldinger, S. D. (1998). Echoes of Echos? An episodic theory of lexical access. Psychological Review, $105,251-279$.

Goldstone, R. L., \& Hendrickson, A. T. (2010). Categorical perception. WIRES Cognitive Science, 1(1), 69-78. https://doi.org/https://doi.org/10.1002/wcs.26

Goldstone, R. L., Lippa, Y., \& Shiffrin, R. M. (2001). Altering object representations through category learning. Cognition, 78, 27-43.

Green, P. A., Brandley, N. C., \& Nowicki, S. (2020). Categorical perception in animal communication and decision-making. Behavioral Ecology, 31(4), 859-867. https://doi.org/10.1093/beheco/araa004

Guenther, F. H., \& Gjaja, M. (1996). The perceptual magnet effect as an emergent property of neural map formation. Journal of the Acoustical Society of America, 100, 1111-1112. 
1727 Guenther, F. H., Nieto-Castanon, A., Ghosh, S., \& Tourville, J. (2004). Representation of Sound

1728

1729

1730

1731

1732

1733

1734

1735

1736

1737

1738

1739

1740

1741

1742

1743

1744

1745

1746

1747

1748

1749

1750

1751

1752

1753

1754

Categories in Auditory Cortical Maps. Journal of Speech Language and Hearing Research, 47, 46-57.

Gwilliams, L., Linzen, T., Poeppel, D., \& Marantz, A. (2018). In Spoken Word Recognition, the Future Predicts the Past. The Journal of Neuroscience, 38(35), 7585-7599. https://doi.org/10.1523/ineurosci.0065-18.2018

Hanley, J. R., \& Roberson, D. (2011). Categorical perception effects reflect differences in typicality on within-category trials. Psychonomic Bulletin \& Review, 18(2), 355-363.

Harnad, S. (2003). Categorical perception.

Hartshorne, J. K., Tenenbaum, J. B., \& Pinker, S. (2018). A critical period for second language acquisition: Evidence from 2/3 million English speakers. Cognition, 177, 263-277. https://doi.org/https://doi.org/10.1016/j.cognition.2018.04.007

Hary, J. M., \& Massaro, D. W. (1982). Categorical results do not imply categorical perception. Perception \& Psychophysics, 32(5), 409-418. https://doi.org/10.3758/BF03202770

Hazan, V., \& Barrett, S. (2000). The development of phonemic categorization in children aged 6-12. Journal of Phonetics, 28(4), 377-396. https://doi.org/http://dx.doi.org/10.1006/ipho.2000.0121

Healy, A. F., \& Repp, B. H. (1982). Context independence and phonetic mediation in categorical perception. Journal of Experimental Psychology: Human Perception and Performance, 8(1), 6880.

Hess, U., Adams, R. B., \& Kleck, R. E. (2009). The Categorical Perception of Emotions and Traits. Social Cognition, 27(2), 320-326. https://doi.org/10.1521/soco.2009.27.2.320

Holt, L. L., \& Lotto, A. J. (2010). Speech perception as categorization. Attention, Perception, \& Psychophysics, 72(5), 1218-1227. https://doi.org/10.3758/APP.72.5.1218

Howard, D., Rosen, S., \& Broad, V. (1992). Major/minor triad identification and discrimination by musically trained and untrained listeners. Music Perception, 10(2), 205-220.

Johnson, J. S., \& Newport, E. L. (1989). Critical period effects in second language learning: The influence of maturational state on the acquisition of English as a second language. Cognitive Psychology, 21(1), 60-99. 
1755 Jongman, A., Wayland, R., \& Wong, S. (2000). Acoustic characteristics of English fricatives. Journal of

1756

1757

1758

1759

1760

1761

1762

1763

1764

1765

1766

1767

1768

1769

1770

1771

1772

1773

1774

1775

1776

1777

1778

1779

1780

1781

1782 the Acoustical Society of America, 106, 1252-1263.

Jusczyk, P. W., \& Aslin, R. N. (1995). Infants' detection of the sound patterns of words in fluent speech. Cognitive Psychology, 29(1), 1-23. https://doi.org/10.1006/cogp.1995.1010

Kapnoula, E. (2015). Individual differences in speech perception: sources, functions, and consequences of phoneme categorization gradiency University of lowa]. lowa City, IA.

Kapnoula, E. C., Edwards, J., \& McMurray, B. (2021). Gradient activation of speech categories facilitates listeners' recovery from lexical garden paths, but not perception of speech-in-noise. Journal of Experimental Psychology: Human Perception and Performance, 47(4). https://psyarxiv.com/hw24k/

Kapnoula, E. C., \& McMurray, B. (2022). Idiosyncratic use of bottom-up and top-down information leads to differences in speech perception flexibility: Converging evidence from ERPs and eye-tracking. Brain and Language, 223, 105031.

Kapnoula, E. C., Winn, M. B., Kong, E., Edwards, J., \& McMurray, B. (2017). Evaluating the sources and functions of gradiency in phoneme categorization: An individual differences approach. Journal of Experimental Psychology: Human Perception and Performance, 43(9), 1594-1611.

Kleinschmidt, D., \& Jaeger, F. (2015). Robust Speech Perception: Recognize the Familiar, Generalize to the Similar, and Adapt to the Novel. Psychological Review, 122(2).

Kluender, K. R., Coady, J., \& Kiefte, M. (2003). Sensitivity to change in perception of speech. Speech Communication, 41(1), 59-69.

Knight, F. L. C., Longo, M. R., \& Bremner, A. J. (2014). Categorical perception of tactile distance. Cognition, 131(2), 254-262. https://doi.org/https://doi.org/10.1016/i.cognition.2014.01.005

Kong, E. J., \& Edwards, J. (2011). Individual differences in speech perception: Evidence from visual analogue scaling and eye-tracking. Proceedings of the XVIIth International Congress of Phonetic Sciences, Hong Kong.

Kronrod, Y., Coppess, E., \& Feldman, N. H. (2016). A unified account of categorical effects in phonetic perception. Psychonomic Bulletin \& Review, 23(6), 1681-1712. https://doi.org/10.3758/s13423$\underline{016-1049-y}$ 
1783 Kuhl, P. K. (1979). Speech perception in early infancy: Perceptual constancy for spectrally dissimilar vowel categories. Journal of the Acoustical Society of America, 66, 1668-1679.

1785 Kuhl, P. K. (2004). Early language acquisition: cracking the speech code [10.1038/nrn1533]. Nat Rev $1786 \quad$ Neurosci, 5(11), 831-843. http://dx.doi.org/10.1038/nrn1533

1787 Kuhl, P. K., \& Miller, J. D. (1975). Speech perception by the chinchilla: Voiced-voiceless distinction in 1788 alveolar plosive consonants. Science, 190(4209), 69-72.

1789 Kuhl, P. K., Stevens, E. H., A., Deguchi, T., Kiritani, S., \& Iverson, P. (2006). Infants show a facilitation 1790 effect for native language phonetic perception between 6 and 12 months. Developmental Science, 9, F13-F21.

Lachlan, R. F., \& Nowicki, S. (2015). Context-dependent categorical perception in a songbird. Proceedings of the National Academy of Sciences, 112(6), 1892-1897. https://doi.org/10.1073/pnas.1410844112

Leopold, D. A., O'Toole, A. J., Vetter, T., \& Blanz, V. (2001). Prototype-referenced shape encoding revealed by high-level aftereffects. Nature Neuroscience, 4(1), 89-94. https://doi.org/10.1038/82947

1798 Levin, D. T., \& Beale, J. M. (2000). Categorical perception occurs in newly learned faces, other-race 1799 faces, and inverted faces. Perception \& Psychophysics, 62(2), 386-401.

1800 Liberman, A. M., Cooper, F. S., Shankweiler, D. P., \& Studdert-Kennedy, M. (1967). Perception of the $1801 \quad$ speech code. Psychological Review, 74(6), 431.

1802 Liberman, A. M., Harris, K. S., Hoffman, H. S., \& Griffith, B. C. (1957). The discrimination of speech 1803 sounds within and across phoneme boundaries. Journal of Experimental Psychology, 54(5), 358368.

Liberman, A. M., Harris, K. S., Kinney, J., \& Lane, H. (1961). The discrimination of relative onset-time of the components of certain speech and non-speech patterns. Journal of Experimental Psychology, 61, 379-388.

Lisker, L. (1986). "Voicing" in English: a catalogue of acoustic features signaling /b/ versus /p/ in trochees. Language and Speech, 29(1), 3-11.

1810 Locke, S., \& Kellar, L. (1973). Categorical perception in a non linguistic mode. Cortex, 9, 355-369. 
1811 Lupyan, G. (2012). Linguistically Modulated Perception and Cognition: The Label-Feedback Hypothesis

1812

1813

1814

1815

1816

1817

1818

1819

1820

1821

1822

1823

1824

1825

1826

1827

1828

1829

1830

1831

1832

1833

1834

1835

1836

1837 [Hypothesis and Theory]. Frontiers in Psychology, 3. https://doi.org/10.3389/fpsyg.2012.00054

Macmillan, N. A., Kaplan, H. L., \& Creelman, C. D. (1977). The psychophysics of categorical perception. Psychological Review, 84(5), 452-471. https://doi.org/10.1037/0033-295X.84.5.452

Mann, V. A., \& Repp, B. (1981). Influence of preceding fricative on stop consonant perception. Journal of the Acoustical Society of America, 69(2), 548-558.

Massaro, D. W., \& Cohen, M. M. (1983). Categorical or continuous speech perception: a new test. Speech Communication, 2, 15-35.

Massaro, D. W., \& Hary, J. M. (1984). Categorical results, categorical perception, and hindsight. Perception \& Psychophysics, 35(6), 586-588.

Maurer, D., \& Werker, J. F. (2014). Perceptual Narrowing During Infancy: A Comparison of Language and Faces. Developmental psychobiology, 56(2), 154-178.

May, B., Moody, D. B., \& Stebbins, W. C. (1989). Categorical perception of conspecific communication sounds by Japanese macaques, M acacafuscata. The Journal of the Acoustical Society of America, 85(2), 837-847.

Maye, J., \& Gerken, L. (2000). Learning phonemes without minimal pairs. Proceedings of the 24th annual Boston university conference on language development,

Maye, J., Weiss, D. J., \& Aslin, R. N. (2008). Statistical phonetic learning in infants: Facilitation and feature generalization. Developmental Science, 11(1), 122-134. https://doi.org/10.1111/j.14677687.2007.00653.x

Maye, J., Werker, J. F., \& Gerken, L. (2003). Infant sensitivity to distributional information can affect phonetic discrimination. Cognition, 82, 101-111.

McClelland, J. L., \& Elman, J. L. (1986). The TRACE model of speech perception. Cognitive Psychology, 18(1), 1-86.

McKone, E., Martini, P., \& Nakayama, K. (2001). Categorical perception of face identity in noise isolates configural processing. Journal of Experimental Psychology: Human Perception and Performance, 27(3), 573. 
1838

1839

McMurray, B. (in press). The acquisition of speech categories: Beyond perceptual narrowing, beyond unsupervised learning and beyond infancy. Language, Cognition and Neuroscience, https://psyarxiv.com/njm3r/.

McMurray, B., \& Aslin, R. N. (2005). Infants are sensitive to within-category variation in speech perception. Cognition, 95(2), B15-B26.

McMurray, B., Aslin, R. N., Tanenhaus, M. K., Spivey, M. J., \& Subik, D. (2008). Gradient sensitivity to within-category variation in words and syllables. Journal of Experimental Psychology, Human Perception and Performance, 34(6), 1609-1631.

McMurray, B., Clayards, M., Tanenhaus, M. K., \& Aslin, R. N. (2008). Tracking the time course of phonetic cue integration during spoken word recognition. Psychonomic Bulletin and Review, 15(6), 1064-1071.

McMurray, B., Danelz, A., Rigler, H., \& Seedorff, M. (2018). Speech categorization develops slowly through adolescence. Developmental Psychology, 54(8), 1472-1491.

McMurray, B., \& Farris-Trimble, A. (2012). Emergent information-level coupling between perception and production. In A. Cohn, C. Fougeron, \& M. Huffman (Eds.), The Oxford Handbook of Laboratory Phonology. The Oxford University Press.

McMurray, B., Farris-Trimble, A., Seedorff, M., \& Rigler, H. (2016). The effect of residual acoustic hearing and adaptation to uncertainty in Cochlear Implant users. Ear and Hearing, 37(1), 37-51.

McMurray, B., \& Jongman, A. (2011). What information is necessary for speech categorization? Harnessing variability in the speech signal by integrating cues computed relative to expectations. Psychological Review, 118(2), 219-246.

McMurray, B., \& Jongman, A. (2015). What comes after [f]? Prediction in speech is a product of expectation and signal. Psychological Science, 27(1), 43-52.

McMurray, B., Munson, C., \& Tomblin, J. B. (2014). Individual differences in language ability are related to variation in word recognition, not speech perception: Evidence from eye-movements. Journal of Speech Language and Hearing Research, 57, 1344-1362.

McMurray, B., Tanenhaus, M. K., \& Aslin, R. N. (2002). Gradient effects of within-category phonetic variation on lexical access. Cognition, 86(2), B33-B42. 
1866

1867

1868

1869

1870

1871

1872

1873

1874

1875

1876

1877

1878

1879

1880

1881

1882

1883

1884

1885

1886

1887

1888

1889

1890

1891

1892

1893

McMurray, B., Tanenhaus, M. K., \& Aslin, R. N. (2009). Within-category VOT affects recovery from "lexical" garden paths: Evidence against phoneme-level inhibition. Journal of Memory and Language, 60(1), 65-91.

Meehl, P. E. (1990). Why Summaries of Research on Psychological Theories are Often Uninterpretable. Psychol Rep, 66(1), 195-244. https://doi.org/10.2466/pr0.1990.66.1.195

Mermelstein, P. (1978). On the relationship between vowel and consonant identification when cued by the same acoustic information. Perception \& Psychophysics, 23, 331-336.

Mesgarani, N., Cheung, C., Johnson, K., \& Chang, E. F. (2014). Phonetic Feature Encoding in Human Superior Temporal Gyrus. Science, 343, 1006-1010.

Miller, J. L. (1997). Internal structure of phonetic categories. Language and Cognitive Processes, 12, 865869.

Miller, J. L., \& Eimas, P. D. (1996). Internal structure of voicing categories in early infancy. Perception \& Psychophysics, 58(8), 1157-1167.

Miller, J. L., \& Volaitis, L. E. (1989). Effect of speaking rate on the perceptual structure of a phonetic category. Perception \& Psychophysics, 46(6), 505-512.

Miyawaki, K., Jenkins, J. J., Strange, W., Liberman, A. M., Verbrugge, R., \& Fujimura, O. (1975). An effect of linguistic experience: The discrimination of [r] and [l] by native speakers of Japanese and English [journal article]. Perception \& Psychophysics, 18(5), 331-340.

\section{https://doi.org/10.3758/bf03211209}

Munson, B. (2007). The acoustic correlates of perceived masculinity, perceived femininity, and perceived sexual orientation. Language and Speech, 50(1), 125-142.

Munson, C. (2011). Perceptual learning in speech reveals pathways of processing. University of lowa]. lowa City, IA.

Myers, E. B., Blumstein, S. E., Walsh, E., \& Eliassen, J. (2009). Inferior Frontal Regions Underlie the Perception of Phonetic Category Invariance. Psychological Science, 20(7), 895-903. https://doi.org/10.1111/j.1467-9280.2009.02380.x

Narayan, C. R., Werker, J. F., \& Beddor, P. S. (2010). The interaction between acoustic salience and language experience in developmental speech perception: evidence from nasal place 
1894

1895

1896

1897

1898

1899

1900

1901

1902

1903

1904

1905

1906

1907

1908

1909

1910

1911

1912

1913

1914

1915

1916

1917

1918

1919

1920

discrimination. Developmental Science, 13(3), 407-420. https://doi.org/10.1111/j.14677687.2009.00898.x

Nearey, T. M. (1997). Speech perception as pattern recognition. Journal of the Acoustical Society of America, 101(6), 3241-3254.

Nearey, T. M., \& Hogan, J. (1986). Phonological contrast in experimental phonetics: Relating distributions of measurements in production data to perceptual categorization curves. In J. Ohala \& J. Jaeger (Eds.), Experimental phonology (pp. 141-161). Academic Press.

Newell, F. N., \& Bülthoff, H. H. (2002). Categorical perception of familiar objects. Cognition, 85(2), 113143.

Nittrouer, S. (1992). Age-related differences in perceptual effects of formant transitions within syllables and across syllable boundaries. Journal of Phonetics.

Nittrouer, S. (2002). Learning to perceive speech: How fricative perception changes, and how it stays the same. Journal of the Acoustical Society of America, 112, 711-719.

Oden, G. (1978). Integration of place and voicing information in the identification of synthetic stop consonants. Journal of Phonetics, 6, 83-93.

Oden, G., \& Massaro, D. W. (1978). Integration of featural information in speech perception. Psychological Review, 85(3), 172-191.

Ohde, R. N. (1984). Fundamental frequency as an acoustic correlate of stop consonant voicing. Journal of the Acoustical Society of America, 75(1), 224-230.

Ou, J., Yu, A. C. L., \& Xiang, M. (2021). Individual Differences in Categorization Gradience As Predicted by Online Processing of Phonetic Cues During Spoken Word Recognition: Evidence From Eye Movements. Cognitive Science, 45(3), e12948. https://doi.org/https://doi.org/10.1111/cogs.12948

Pasley, B. N., David, S. V., Mesgarani, N., Flinker, A., Shamma, S. A., Crone, N. E., Knight, R. T., \& Chang, E. F. (2012). Reconstructing Speech from Human Auditory Cortex. PLoS Biology, 10(1), e1001251. https://doi.org/10.1371/journal.pbio.1001251

Phillips, C. (2001). Levels of representation in the electrophysiology of speech perception. Cognitive Science, 25(5), 711-731. https://doi.org/10.1207/s15516709cog2505 5 
1921 Pisoni, D. B. (1973). Auditory and phonetic memory codes in the discrimination of consonants and

1922

1923

1924

1925

1926

1927

1928

1929

1930

1931

1932

1933

1934

1935

1936

1937

1938

1939

1940

1941

1942

1943

1944

1945

1946

1947

1948 vowels. Perception \& Psychophysics, 13(2), 253-260. https://doi.org/10.3758/BF03214136

Pisoni, D. B. (1977). Identification and discrimination of the relative onset time of two component tones: Implications for voicing perception in stops. The Journal of the Acoustical Society of America, 61(5), 1352-1361. https://doi.org/10.1121/1.381409

Pisoni, D. B., \& Lazarus, J. H. (1974). Categorical and noncategorical modes of speech perception along the voicing continuum. Journal of the Acoustical Society of America, 55, 328-333.

Pisoni, D. B., \& Tash, J. (1974). Reaction times to comparisons within and across phonetic categories. Perception \& Psychophysics, 15(2), 285-290.

Plichta, B., \& Preston, D. R. (2005). The /ay/s have It the perception of /ay/ as a north-south stereotype in United States English. Acta Linguistica Hafniensia, 37(1), 107-130. https://doi.org/10.1080/03740463.2005.10416086

Pollack, I., \& Pisoni, D. B. (1971). On the comparison between identification and discrimination tests in speech perception. Psychonomic Science, 24(6), 299-300.

Quinn, P. C. (2004). Visual perception of orientation is categorical near vertical and continuous near horizontal. Perception, 33(8), 897-906.

Rao, V. N. V., Bye, J. K., \& Varma, S. (2021). Categorical Perception of p-Values. Topics in Cognitive Science. https://doi.org/https://doi.org/10.1111/tops.12589

Reinisch, E., \& Sjerps, M. J. (2013). The uptake of spectral and temporal cues in vowel perception is rapidly influenced by context. Journal of Phonetics, 41(2), 101-116.

Repp, B. (1984). Categorical perception: Issues, methods and findings. In N. Lass (Ed.), Speech and Language (vol. 10): Advances in Basic Research and Practice (pp. 244-335). Academic Press.

Repp, B. H. (1982). Phonetic trading relations and context effects: New experimental evidence for a speech mode of perception. Psychological Bulletin, 92(1), 81.

Rhodes, G., \& Leopold, D. A. (2011). Adaptive norm-based coding of face identity. The Oxford handbook of face perception, 263-286.

Roberson, D., Davies, I., \& Davidoff, J. (2000). Color categories are not universal: replications and new evidence from a stone-age culture. Journal of Experimental Psychology: General, 129(3), 369. 
1949

1950

1951

1952

1953

1954

1955

1956

1957

1958

1959

1960

1961

1962

1963

1964

1965

1966

1967

1968

1969

1970

1971

1972

1973

1974

1975

1976

Roberson, D., Hanley, J. R., \& Pak, H. (2009). Thresholds for color discrimination in English and Korean speakers. Cognition, 112(3), 482-487.

Robertson, E. K., Joanisse, M. F., Desroches, A. S., \& Ng, S. (2009). Categorical speech perception deficits distinguish language and reading impairments in children. Developmental Science, 12(5), 753-767.

Rosen, S. M. (1979). Range and frequency effects in consonant categorization. Journal of Phonetics, 7(4), 393-402. https://doi.org/https://doi.org/10.1016/S0095-4470(19)31072-1

Rosen, S. M., \& Howell, P. (1981). Plucks and bows are not categorically perceived. Perception \& Psychophysics, 30(2), 156-168. https://doi.org/10.3758/BF03204474

Sarrett, M., McMurray, B., \& Kapnoula, E. (2020). Dynamic EEG analysis during language comprehension reveals interactive cascades between perceptual processing and semantic expectations. Brain and Language, 211, 104875.

Schatz, T., Feldman, N. H., Goldwater, S., Cao, X.-N., \& Dupoux, E. (2021). Early phonetic learning without phonetic categories: Insights from large-scale simulations on realistic input. Proceedings of the National Academy of Sciences, 118(7), e2001844118. https://doi.org/doi:10.1073/pnas.2001844118

Schouten, M. E. H., Gerrits, E., \& Van Hessen, A. (2003). The end of categorical perception as we know it. Speech Communication, 41, 71-80.

Serniclaes, W., Van Heghe, S., Mousty, P., Carre, R., \& Sprenger-Charolle, L. (2004). Allophonic mode of speech perception in dyslexia. Journal of Experimental Child Psychology, 87, 336-361.

Skinner, B. F. (1957). Verbal Behavior. Copley Publishing Group. https://doi.org/10.1037/11256-000

Slawinski, E. B., \& Fitzgerald, L. K. (1998). Perceptual development of the categorization of the/r-w/ contrast in normal children. Journal of Phonetics, 26, 27-43.

Smits, R. (2001). Hierarchical categorization of coarticulated phonemes: A theoretical analysis. Perception \& Psychophysics, 63, 1109-1139.

Steinschneider, M., Volkov, I. O., Noh, M. D., Garell, P. C., \& Howard, M. A. (1999). Temporal Encoding of the Voice Onset Time Phonetic Parameter by Field Potentials Recorded Directly From Human Auditory Cortex (Vol. 82) [Journal Article]. http://jn.physiology.org/in/82/5/2346.full.pdf 
1977 1978 1979 1980 1981

Strand, E. (1999). Uncovering the Role of Gender Stereotypes in Speech Perception. Journal of Language and Social Psychology, 18, 86-100.

Strange, W., \& Shafer, V. L. (2008). Speech perception in second langauge learners: The re-education of selective perception. In J. G. H. Hansen \& M. Zampini (Eds.), Phonology and Second Language Acquisition (pp. 153-192). John Benjamins.

Szostak, C. M., \& Pitt, M. A. (2013). The prolonged influence of subsequent context on spoken word recognition [journal article]. Attention, Perception, \& Psychophysics, 75(7), 1533-1546. https://doi.org/10.3758/s13414-013-0492-3

Theodore, R. M., \& Monto, N. R. (2019). Distributional learning for speech reflects cumulative exposure to a talker's phonetic distributions. Psychonomic Bulletin \& Review, 26(3), 985-992. https://doi.org/10.3758/s13423-018-1551-5

Thiessen, E. D., Girard, S., \& Erickson, L. C. (2016). Statistical learning and the critical period: how a continuous learning mechanism can give rise to discontinuous learning. WIRES Cognitive Science, 7(4), 276-288. https://doi.org/https://doi.org/10.1002/wcs.1394

Toscano, J. C., Anderson, N. D., Fabiani, M., Gratton, G., \& Garnsey, S. M. (2018). The time-course of cortical responses to speech revealed by fast optical imaging. Brain and Language, 184, 32-42. https://doi.org/https://doi.org/10.1016/j.bandl.2018.06.006

Toscano, J. C., \& McMurray, B. (2010). Cue integration with categories: Weighting acoustic cues in speech using unsupervised learning and distributional statistics. Cognitive Science, 34(3), 436464.

Toscano, J. C., \& McMurray, B. (2015). The time-course of speaking rate compensation: Effects of sentential rate and vowel length on voicing judgments. Language, Cognition and Neuroscience, $30,529-543$.

Toscano, J. C., McMurray, B., Dennhardt, J., \& Luck, S. (2010). Continuous Perception and Graded Categorization Electrophysiological Evidence for a Linear Relationship Between the Acoustic Signal and Perceptual Encoding of Speech. Psychological Science, 21(10), 1532-1540.

Tsuji, S., \& Cristia, A. (2014). Perceptual attunement in vowels: A meta-analysis. Developmental psychobiology, 56(179-191). 
2005

2006

2007

2008

2009

2010

2011

2012

2013

2014

2015

2016

2017

2018

2019

2020

2021

2022

2023

2024

2025

2026

2027

2028

2029

2030

2031

Utman, J. A., Blumstein, S. E., \& Burton, M. W. (2000). Effects of subphonetic and syllable structure variation on word recognition. Perception \& Psychophysics, 62(6), 1297-1311.

Van Hessen, A. J., \& Schouten, M. E. H. (1999). Categorical Perception as a Function of Stimulus Quality. Phonetica, 56, 56-72.

Werker, J. F., \& Curtin, S. (2005). PRIMIR: A Developmental Framework of Infant Speech Processing. Language Learning and Development, 1(2), 197-234. https://doi.org/10.1080/15475441.2005.9684216

Werker, J. F., \& Hensch, T. K. (2015). Critical periods in speech perception: new directions. Annual Review of Psychology, 66(1), 173-196.

Werker, J. F., \& Tees, R. C. (1984). Cross-language speech perception: Evidence for perceptual reorganization during the first year of life. Infant Behavior and Development, 7, 49-63.

Werker, J. F., \& Tees, R. C. (1987). Speech perception in severely disabled and average reading children. Canadian Journal of Psychology, 41(1), 48-61.

Werker, J. F., \& Yeung, H. H. (2005). Infant speech perception bootstraps word learning. Trends in Cognitive Sciences, 9(11), 519-527. https://doi.org/https://doi.org/10.1016/j.tics.2005.09.003

Whalen, D. H. (1992). Perception of overlapping segments: Thoughts on Nearey's model. Journal of Phonetics, 20, 493-496.

Wyttenbach, R. A., May, M. L., \& Hoy, R. R. (1996). Categorical Perception of Sound Frequency by Crickets. Science, 273(5281), 1542-1544. https://doi.org/doi:10.1126/science.273.5281.1542

Yeni-Komshian, G. H., \& Soli, S. D. (1981). Recognition of vowels from information in fricatives: Perceptual evidence of fricative-vowel coarticulation. The Journal of the Acoustical Society of America, 70, 966.

Zhang, Q., Lei, L., \& Gong, T. (2021). Categorical Perception as a Combination of Nature and Nurture. . Annual Meeting of the Cognitive Science Society 43,

Zimman, L. (2017). Variability in/s/among transgender speakers: Evidence for a socially grounded account of gender and sibilants. Linguistics, 55(5), 993-1019. 


\title{
The Myth of Categorical Perception
}

ONLINE SUPPLEMENT

\author{
Bob McMurray \\ Dept. of Psychological and Brain Sciences \\ Dept. of Linguistics \\ Dept. of Communication Sciences and Disorders \\ University of lowa \\ and \\ Haskins Laboratories
}

Contact

Bob McMurray

278 PBSB

Dept. of Psychological and Brain Sciences

University of lowa

lowa City, IA 52242

319-335-2408

Bob-mcmurray@uiowa.edu 


\section{S1: Standardizing Speech Continua}

Despite decades of work using speech continua there are no attempts to standardize how these are reported or used across studies. This is a challenging problem for many reasons. First, the acoustic dimensions are often incommensurate - how many msec of VOT equals a $20 \mathrm{~Hz}$ shift in F0? Second, the approach to continuum generation is highly variable: some approaches would vary a single cue (Klatt, 1980), while others "morph" one natural recording into another (Kawahara et al., 1999).

However, standardizing how these manipulations are reported could yield two benefits. First, it could help understand similar effects applied across different speech sounds. For example, some perceptual learning effects appear to work differently across different speech contrasts (e.g., voicing vs. fricative place). However, without a standard unit these cannot really be compared. Second, it would allow rigorous quantitative standards-such as how big of a boundary shift is meaningful, or how steep is a slope-that can be applied across papers. Both are essential for meta-analyses.

One way to solve this problem is to rely on the phonetic measurements. If we assume that speech contrasts are coded as prototypes (not as a "boundary"), we know what a /b/ and a /p/ look like. We can measure a large set of cues for $\mathrm{a} / \mathrm{b} / \mathrm{in}$ a set of recordings or in a corpus and use these to scale the continuum steps in terms of a proportion of the distance between the two prototypes.

Here, I work out an example of how this would work. I do this for several progressively more complex cases that range from a simple case of synthesizing a continuum along a single cue, to one that uses a more naturalistic morphing process (ala STRAIGHT).

\section{S1.1 A single cue continuum}

In this example, we consider constructing a VOT continuum for English. Typical approaches would start from $0 \mathrm{msec}$ (canonically a /b/) and vary in $10 \mathrm{msec}$ steps to maybe $50 \mathrm{msec}(\mathrm{a} / \mathrm{p} /$ ) (l've kept the continuum small to ease exposition). Let's presume one has already made this continuum. How can this be standardized?

First, start by recording some tokens of $/ \mathrm{b} /$ and $/ \mathrm{p} /$. Since VOT is affected by the neighboring vowel, talker, speaking rate etc. these things should be roughly matched to your stimulus (e.g., use the same word, the same gender of the talker). If one is doing a form of copy synthesis (e.g., based on original recordings), one should use the same talker. However, even in this case, since individual tokens will vary from utterance to utterance, the experimenter should record multiple tokens of each endpoint.

Second, measure the VOT (or whatever cue is being manipulated) and compute the average for the endpoints. That becomes your prototype for each category.

Third, measure the VOT of each continuum member (if not already known from the synthesis parameters).

Finally, recompute the VOTs that were present in the continuum as the distance between the endpoints using equation 1.

$$
\text { Step }=\left(\mathrm{VOT}_{\text {actual }}-\mathrm{VOT}_{\mathrm{B}}\right) /\left(\mathrm{VOT}_{\mathrm{P}}-\mathrm{VOT}_{\mathrm{B}}\right)
$$


For various assumptions, this would give something like the table below.

\begin{tabular}{|c|c|c|c|c|c|c|c|}
\hline $\begin{array}{r}\text { Fit of continuum to } \\
\text { prototypes }\end{array}$ & $\begin{array}{r}\text { Original } \\
\text { VOT }\end{array}$ & 0 & 10 & 20 & 30 & 40 & 50 \\
\hline Match & $\begin{array}{r}\text { B: } 0 \mathrm{msec} \\
\text { P: } 50 \mathrm{msec}\end{array}$ & .00 & .20 & .40 & 60 & .80 & 1.00 \\
\hline $\begin{array}{l}\text { Cont } \\
\text { protc }\end{array}$ & $\begin{array}{l}\text { B: }-3 \mathrm{msec} \\
\text { P: } 58 \mathrm{msec}\end{array}$ & 049 & .213 & .377 & .541 & .705 & .8 \\
\hline $\begin{array}{l}\text { Continuum extends } \\
\text { beyond prototypes }\end{array}$ & $\begin{array}{l}\text { B: } 5 \mathrm{msec} \\
\text { P: } 45 \mathrm{msec}\end{array}$ & -.125 & .125 & .375 & .625 & .875 & 1.1 \\
\hline
\end{tabular}

This works equally well for a log scaled continuum (which is common for many temporal cues or frequency cues that are in the lower end of the scale such as F0 or F1). Here, the mapping from the original VOT to the proportion is still linear (to aid interpretation across studies, but the underlying continuum is logarithmic.

\begin{tabular}{|c|c|c|c|c|c|c|c|}
\hline $\begin{array}{r}\text { Fit of continuum to } \\
\text { prototypes }\end{array}$ & $\begin{array}{c}\text { Original } \\
\text { VOT }\end{array}$ & 0 & 1.2 & 3.84 & 9.65 & 22.42 & 50.5 \\
\hline Match & $\begin{array}{r}\text { B: } 0 \mathrm{msec} \\
\mathrm{P}: 50 \mathrm{msec}\end{array}$ & .00 & .024 & .077 & .193 & .448 & 1.01 \\
\hline $\begin{array}{l}\text { Continuum inside } \\
\text { prototypes }\end{array}$ & $\begin{array}{l}\text { B: }-3 \mathrm{msec} \\
\mathrm{P}: 58 \mathrm{msec}\end{array}$ & .049 & .069 & .112 & .207 & .416 & .877 \\
\hline $\begin{array}{l}\text { Continuum extends } \\
\text { beyond prototypes }\end{array}$ & $\begin{array}{l}\text { B: } 5 \mathrm{msec} \\
\mathrm{P}: 45 \mathrm{msec}\end{array}$ & -.125 & -.095 & -.029 & .116 & .436 & 1.138 \\
\hline
\end{tabular}

\section{S1.2 Multiple related continua}

What about the case in which there are multiple VOT continua which vary in factors that may affect the prototypes? For example, consider an experiment which tested beach/peach and back/pack. Here the height of the vowel could affect the VOT. Or consider an experiment with a beach/peach continuum, but spoken by a male and female talker.

One should start by recording (as usual) multiple exemplars for each word and measuring the corresponding VOT. At this point there are two options. First, one could use slightly different prototypes for each continuum. This will result in different steps for each continuum. It may make it difficult to plot, but statistical analysis should not be problematic, since logistic mixed models can treat step as a continuous variable. Alternatively, one could average the prototypes and apply the same prototypes to both.

How one chooses to do this may matter. For example, if the goal was to capture differences between the two continua (e.g., do males have a different boundary than females), the first option will not work: by normalizing each continuum to separate male or female prototype values, you would be eliminating the differences you want to study. Here, using pooled estimates would make the most sense. Alternatively, if this is nuisance variance (you don't care about these differences), then separate prototypes would help control this and give you a more precise estimate of where each step lay along that continuum.

\section{S1.3 Multiple cues in a continuum}


Other times, one might simultaneously vary two cues, for example VOT and F0. Similarly, algorithms like STRAIGHT may simultaneously manipulate dozens of cues as it essentially morphs between two recordings.

Conceptually, we can use the same general procedure, but we have to do a little linear algebra. First, we need to identify each token of the continuum in a multi-dimensional cue space. For the VOT/FO example, that's easy - those were decided in advance. For a case like STRAIGHT, one would need to identify the relevant cues and measure them for each token.

Next, we convert each of those cues to its relative location along each dimension using the formula defined above.

\begin{tabular}{|l|r|c|c|c|c|c|c|}
\hline \multirow{2}{*}{ Original Values } & $\mathbf{V O T}$ & $\mathbf{0}$ & $\mathbf{1 0}$ & $\mathbf{2 0}$ & $\mathbf{3 0}$ & $\mathbf{4 0}$ & $\mathbf{5 0}$ \\
\cline { 2 - 8 } & $\mathbf{F 0}$ & 140 & 144 & 148 & 152 & 156 & 160 \\
\hline $\begin{array}{l}\text { Prototypes (from } \\
\text { phonetic data) }\end{array}$ & $\mathbf{V O T}$ & -3 & & & & & 58 \\
\hline \multirow{2}{*}{ Relative Location } & VOT & 135 & & & & & 150 \\
\cline { 2 - 8 } & $\mathbf{F 0}$ & .2 & .213 & .377 & .541 & .705 & .868 \\
\hline & Combined & .124 & .287 & .449 & .610 & .772 & .934 \\
\hline
\end{tabular}

In this relative space, we can now visualize the prototypes located at $\langle 0,0\rangle(/ \mathrm{b} /)$, and $\langle 1,1\rangle$ $(/ \mathrm{p} /)$. Each token has a coordinate in that space. The first step of the continuum, for example is at .049 on the VOT dimension, and 0.20 on the F0 dimension.

Next, we simply need to project that token onto its closest point along the diagonal. That will give us the relative location of that point between the two prototypes. This can be done with a little linear algebra (but actually, it turns out that you don't).

Start from a vector notation in which $\vec{s}$ stores the coordinates of that step $\left(\begin{array}{c}V O T \\ F 0\end{array}\right)$. We need to project that point onto the closest point of a line defined by the vector, $\vec{v}$. Since we've relativized the distance, this vector is $\left(\begin{array}{l}1 \\ 1\end{array}\right)$. We now apply the standard formula for projecting $\vec{s}$ onto $\vec{v}$

$$
\operatorname{projection}_{\vec{s}}(\vec{v})=\frac{\vec{s} \cdot \vec{v}}{\vec{v} \cdot \vec{v}} \cdot \vec{v}
$$

However, since $\cdot \vec{v}=\left(\begin{array}{l}1 \\ 1\end{array}\right)$ this equation simplifies dramatically: $\vec{v} \cdot \vec{v}=2$, and since the first half of the equation will be a constant (not a vector), multiplying it by $\left(\begin{array}{l}1 \\ 1\end{array}\right)$ does nothing. Moreover, the dot product of $\vec{s} \cdot\left(\begin{array}{l}1 \\ 1\end{array}\right)$ is the sum of the elements of $\vec{s}$. As a result the coordinates of the projection are now the sum of the two relative cues divided by two-the average! This can be done with any number of cues.

\section{S.1.4 Summary}

The procedures explained here share the goal of locating each step of a continuum along a line defined by two endpoints that are grounded in phonetic measurements of a real set of utterances. That is, $20 \mathrm{~Hz}$ of formant frequency change may be incommensurate with $5 \mathrm{msec}$ of VOT; however, by converting both to $20 \%$ of the difference between the two endpoints, we now have a common scale. Adopting such procedures uniformly could help standardize and compare effects across different continua, and it could lead to standards (how big of a boundary shift is meaningful). 
Critically, if one adopts this procedure, one should report (in a supplement) the details of the corpora on which the prototypes are based, the mean and variance of the prototypes, the specific prototypes used for each continuum, and both the raw (measured) continuum steps and the relative ones. 


\section{S2. Informal Observations about the History of Speech Perception}

Categorical perception predates my birth by almost a decade. So, it is hardly my place to comment on the nature of the field at the time. And yet, l've been trained by folks who were there; I've [politely] fought with some them; and l've closely read much of that seminal work in a way that is not always done today. At the same time, l've also been involved in modern approaches that embrace more naturalistic samples, big datasets and sophisticated modeling, that admit more variability in speakers and listeners (though many younger readers may argue I don't go far enough), and that take advantage of much richer and more sophisticated phonetic analyses. So, as a sort of bridge, l'm in an interesting position to comment (classic Generation $\mathrm{X}$, I suppose).

Figure S1 shows a photograph I took on my last visit to the Haskins laboratory. On the wall of their historical archives are original printouts ${ }^{1}$ from Al Liberman and Franklin Cooper's seminal investigation of the phonetics of consonants (Delattre et al., 1955). Somewhat amazing is the breadth - every consonant of English in every vowel context. These are people who deeply appreciated what's in the signal and the way it varies across utterances.

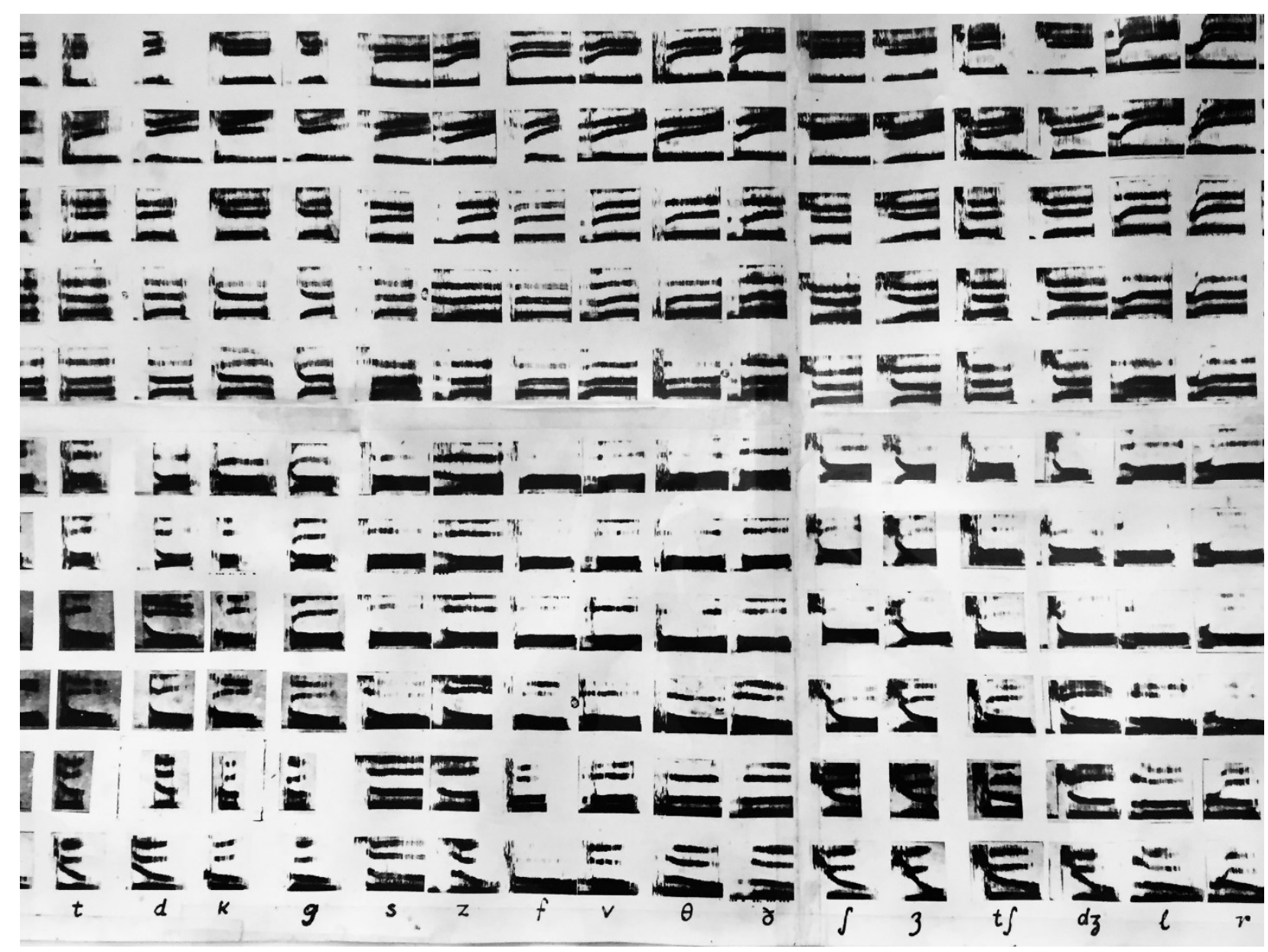

Figure S1: Photographs of the original sound spectrograms posted on the wall of the Haskins Laboratory.

${ }^{1}$ Actually, "printout" is not the right word as it implies that there is some copy (digital or otherwise) that is reproduced on paper; at the time, this would have been the only available rendering of the spectrogram. 
But at the same time, the more modern scientist in me can't help but notice what's missing. These are all spoken by the same person. They are all in a single syllabic frame (CV), and all spoken in isolation. Only three formants are present in most, and other cues that now know are important (e.g., duration, bandwidth, nasal zeros) may be difficult to measure. There is only one recording of each utterance. There was no way to pool these images for any kind of sophisticated statistical analysis. And the pattern playback generated utterances used to tested these hypotheses are even simpler.

If this is where you were coming from, you can see the attraction of CP. However, if we had approached the problem with fresh eyes - appreciating the enormous variation of speech, the variable functions it must serve (phonological, sociolinguistic and others), and with the tools of big data would we have ever reached the same conclusion? It's not clear to me that we would have.

\section{References}

Delattre, P., Liberman, A. M., \& Cooper, F. S. (1955). Acoustic loci and transitional cues for consonants. Journal of the Acoustical Society of America, 27, 769-773.

Kawahara, H., Masuda-Katsuse, I., \& de Cheveigné, A. (1999). Restructuring speech representations using a pitch-adaptive time-frequency smoothing and an instantaneousfrequency-based F0 extraction: Possible role of a repetitive structure in sounds. Speech Communication, 27(3), 187-207. https://doi.org/http://dx.doi.org/10.1016/S01676393(98)00085-5

Klatt, D. (1980). Software for a Cascade/Parallel Synthesizer. Journal of the Acoustical Society of America, 67, 971-995. 